\title{
Implementación de un sistema integrado de planificación de recursos empresariales para mejorar la productividad en las recaudaciones por caja de una importante clínica de la ciudad de Lima
}

\begin{abstract}
RESUMEN
El presente trabajo de investigación tiene por finalidad demostrar los resultados obtenidos a partir de la implementación de un sistema integrado de planificación de recursos empresariales en una importante clínica de la ciudad de Lima. Para determinar las mejoras 0 beneficios de la implementación, se aplicaron técnicas cuantitativas y cualitativas, cuyos resultados se muestran en cuadros y gráficos elaborados con base en la información obtenida de los usuarios del sistema ERP/módulo de caja. Se concluye que un mejor control de las recaudaciones por caja contribuye a un mejor rendimiento en la productividad y una mejor atención en el servicio.
\end{abstract}

Palabras clave: Sistema ERP; sistema Msante; automatización; SUNAT; control.

\section{INTRODUCCIÓN}

Los negocios necesitan estar alineados con los sistemas de información para lograr innovación, competitividad y un mejor posicionamiento en el mercado. Esto es posible a través de procesos y actividades ágiles que permiten eficiencia y eficacia en las operaciones, lo que resulta en que se obtenga información óptima para una buena toma de decisiones y mejoras continuas.

El presente trabajo de investigación expone el desarrollo en el conocimiento de los sistemas de planificación de recursos empresariales, conocidos como ERP por sus siglas en inglés, implementados en una clínica de la ciudad de Lima; asimismo, muestra la forma en que estos sistemas contribuyen (1) en la labor que desempeña cada colaborador o usuario, al ser una herramienta de trabajo sencilla y agradable para el buen desenvolvimiento de las tareas asignadas; y (2) en las mejoras productivas de la clínica a través de la integración de la información.

Al observar y analizar el sistema de planificación de recursos empresariales implementado en la clínica, se hace evidente que este permite planificar cada actividad de forma ordenada, respetando los procedimientos establecidos; mejora el flujo documentario; y optimiza los tiempos, de modo que se cumple con los requisitos de entes fiscalizadores, tales como la SUNAT. Así, se obtiene el control del proceso de caja, lo que trasciende en los resultados de las cobranzas.

Los sistemas ERP fueron creados para empresas que manejan inventarios de existencias o de manufacturas; sin embargo, la novedad de este estudio radica en que se demuestra que este sistema también es aplicable a una empresa netamente de servicios. Asimismo, este estudio permitirá ampliar el conocimiento que se tiene sobre el sistema ERP al plantear su aplicabilidad en una empresa del sector de servicios de salud; esta aplicabilidad se

1 Contador público por la Universidad Nacional del Callao (Lima, Perú). Actualmente, es docente del Instituto Certus (Lima,Perú).

ORCID: https://orcid.org/0000-0002-5384-718X

Autor de correspondencia: jinfantesl@certus.edu.pe

2 Licenciado en Computación por la UNMSM (Lima, Perú). Actualmente, es docente asociado de la UNMSM (Lima, Perú).

ORCID: https://orcid.org/0000-0001-9531-881X

E-mail: smoquillazah@unmsm.edu.pe 
IMPLEMENTACIÓN DE UN SISTEMA INTEGRADO DE PLANIFICACIÓN DE RECURSOS EMPRESARIALES PARA MEJORAR LA PRODUCTIVIDAD EN LAS RECAUDACIONES POR CAJA DE UNA IMPORTANTE CLIIIICA DE LA CIUDAD DE LIMA

demuestra por medio del procesamiento de la información provista por los usuarios del módulo de caja de la clínica sobre los beneficios que se obtienen.

Según Duke, Navarro, Díaz, Pérez, y Vargas-Lombardo (2016), "los principales retos que presentan las organizaciones hoy, es enfrentar los constantes cambios al mercado actual, con la necesidad de implementar sistemas que ayuden a la buena comunicación e integración entre las distintas áreas de una organización" (p. 87).

Surge entonces la necesidad de ordenar actividades y procesos de manera integrada, es decir, es necesario que cada departamento, división o área de cada negocio mantenga siempre una comunicación fluida y acertada y que, a la vez, cada parte operativa interactúe con cada unidad de la organización en busca de un mejor control que permita las decisiones tomadas estén respaldadas por información correcta.

Indudablemente, el buen funcionamiento del sistema ERP dependerá de una adecuada configuración $y$, en parte, de la predisposición del personal, así como de su experiencia. Dado el costo de inversión que requiere este tipo de sistema, no cabe duda de que los beneficios se obtendrán a mediano y largo plazo. Por ello, se deben mejorar aspectos sobre el procesamiento o traslado adecuado de la información, de modo que el área pertinente o especializada cuente con los datos correctos; de lo contrario, la información provista no será precisa.

Por lo expuesto líneas arriba, se planteó como objetivo general "implementar un sistema ERP para mejorar el control de las recaudaciones por caja en una importante clínica ubicada en la ciudad de Lima". Asimismo, los objetivos específicos fueron "demostrar que la automatización de operaciones por medio del sistema Spring ERP/módulo de caja contribuirá con el control de las recaudaciones por caja de una importante clínica ubicada en la ciudad de Lima", "corroborar que la mejora del proceso de información por medio del sistema Spring ERP/módulo de caja acrecentará el control de las recaudaciones por caja de una importante clínica ubicada en la ciudad de Lima" y "comprobar que los arqueos de caja por medio del sistema Spring ERP/módulo de caja generarán un adecuado control de las recaudaciones por caja de una importante clínica ubicada en la ciudad de Lima".

Una ventaja que del sistema ERP es que contribuye con el cumplimiento de los objetivos por medio de la parametrización.
Tal como lo define Suárez (2016):

La parametrización de un ERP permite adaptar el funcionamiento del sistema a las necesidades concretas de cada empresa, así como incorporar nuevas funciones o modos de funcionamiento a medida que la empresa en cuestión lo requiere, sin requerir de desarrollos específicos o a medida del cliente [sic]. (p. 25)

\section{HIPÓTESIS GENERAL}

La implementación de un sistema ERP mejora el control de las recaudaciones por caja en una importante clínica ubicada en la ciudad de Lima.

\section{HIPÓTESIS ESPECÍFICAS}

1. La automatización de operaciones por medio del sistema Spring ERP/módulo de caja contribuirá con el control de las recaudaciones por caja en una importante clínica ubicada en la ciudad de Lima.

2. La mejora del proceso de información por medio del sistema Spring ERP/módulo de caja acrecentará el control de las recaudaciones por caja en una importante clínica ubicada en la ciudad de Lima.

3. Los arqueos de caja por medio del sistema Spring ERP/módulo de caja generarán un adecuado control de las recaudaciones por caja en una importante clínica ubicada en la ciudad de Lima.

\section{JUSTIFICACIÓN}

Para Hernández, Carreño, Sandoval, Estrada e Ignacio (2016), "desde hace más de tres décadas los Sistemas Enterprise Resource Planning (ERP) en inglés, conocidos como Sistemas de Planificación de Recursos, Empresariales (sic), han otorgado alta competitividad al mundo empresarial" ( $p$. 234). Con base en lo citado, la implementación de un sistema ERP en una importante clínica de la ciudad de Lima se hizo necesaria para la mejora de los procesos que contribuyen en el control de las recaudaciones o cobranzas por caja a través del módulo de caja. Asimismo, esta implementación contribuiría en la mejora de los procedimientos de atención e información al cliente o paciente y lograría eliminar procedimientos manuales para facilitar las labores de caja, brindar información correcta y optimizar las actividades y tareas del 
área contable, de modo que esta maneje una correcta informacion financiera.

Según Farfán (2020), “a través de la automatización de la gestión por procesos, las organizaciones pueden tener un mejor funcionamiento, aumentando la eficiencia de las mismas, lo cual representa una ventaja competitiva" (p. 36).

El sistema de información permite diseñar mejores estructuras informáticas amigables para una buena revisión y supervisión de las operaciones, lo que permite que la parte contable pueda verificar cada operación y que se cumplan con las normas del ente fiscalizador y de control.

\section{Según Fernández (2015):}

El arqueo de caja "consiste en la realización de una [sic] análisis de la [sic] transacciones de efectivo que se han producido en al [sic] empresa en un lapso de tiempo determinado". La finalidad del mismo es comprobar si existen diferencias entre el efectivo contabilizado y el recuento de billetes, monedas y cheques físicamente que tenemos en nuestra caja. Hemos de tener en cuenta que todo registro contable debe estar soportado documentalmente, de modo que cualquier error u omisión debe ser fácilmente detectable. (p. 94)

Durante el arqueo de caja se ejecuta la contrastación y control de la documentación, lo que permite contabilizar con exactitud los diferentes medios de pago. Al incluir el arqueo de caja en un sistema ERP, se busca cumplir con la bancarización y con el pago del impuesto a las transacciones financieras (ITF), según lo dispuesto por la Ley 28194, vigente desde el año 2004.

Mayorga, Espinosa, López y Chango (2020) indican que "los sistemas de información permiten a la organización captar, identificar y comunicar de forma adecuada el plazo permitido al personal para afrontar sus responsabilidades. La comunicación debe ser fluida en todo el conjunto de la institución" (p. 62).

Según Hurtado, Arroyo y Guzmán (2019), "el control interno puede ayudar a que una entidad consiga sus objetivos de rentabilidad y rendimiento y a prevenir la pérdida de recursos" (p. 6).

La implementación del sistema ERP ha logrado mantener en un puesto de vanguardia a una importante clínica privada de la ciudad de Lima, que tiene una trayectoria de más de 150 años y que cuenta con tres sedes en los distritos de Lima, Chorrillos y Surco.

\section{SISTEMAS DE PLANIFICACIÓN DE RECUR- SOS EMPRESARIALES (ERP)}

Según Riascos y Arias (2016), "la decisión de implementar un sistema de información ERP le facilita a la organización desarrollar sus procesos administrativos de forma eficiente y eficaz" (p. 289).

El sistema ERP es un programa adaptable que da resultados óptimos de gestión y de operatividad, puesto que permite aprovechar al máximo los recursos que predispone de forma integrada. Este sistema permite configurar o moldear las diversas labores de manera estructurada de acuerdo con el perfil del usuario; de esta forma, proporciona información confiable que es respaldada por una plataforma de base de datos.

Así, Núñez (2016) señala:

Típicamente, un sistema ERP es un sistema de información integrado en la forma de un paquete de software compuesto por varios módulos, tales como producción, ventas, finanzas y recursos humanos, que nos aporta una integración de datos horizontales a lo largo de la organización y a través de sus procesos de negocio. Esos paquetes pueden ser personalizados de forma que respondan a las necesidades específicas de la organización. (p. 7)

Por otra parte, Oliva, Ortigoza y Méndez señalan que "Los sistemas ERP son soluciones de software de gestión de información que se ocupa de las necesidades de las empresas de manera integral a través de la automatización de procesos operativos" (2012, p. 2, como se citó en Guerrero, Marín y Bonilla, 2018, p. 185).

Según Suárez (2016), "podemos definir un sistema ERP como un software integrado de gestión empresarial, compuesto por un conjunto de módulos funcionales (logística, finanzas, recursos humanos, etc.) susceptibles de ser adaptados a las necesidades de cada cliente" (p. 19).

Un sistema ERP logra la automatización cuando, mediante este, las actividades se realizan de manera sencilla en el menor tiempo posible; sin embargo, si las actividades y procesos no son claros, los resultados serán los mismos. 
IMPLEMENTACIÓN DE UN SISTEMA INTEGRADO DE PLANIFICACIÓN DE RECURSOS EMPRESARIALES PARA MEJORAR LA PRODUCTIVIDAD EN LAS RECAUDACIONES POR CAJA DE UNA IMPORTANTE CLIIIICA DE LA CIUDAD DE LIMA

Es indispensable considerar al área contable dentro de un sistema ERP, puesto que se trata de un área donde se centraliza la información. Así, la participación contable es imprescindible en cada área o unidad de negocio, puesto que contribuye en la mejora de los diseños o estructuras de operatividad; así facilita la fluidez de la información contable, de modo que esta sea confiable.

De acuerdo con lo publicado en ESAN (14 de setiembre de 2018), "el sistema de planificación de recursos empresariales o enterprise resource planning (ERP) es un software desarrollado para gestionar los principales procesos empresariales".

Según Hernández, Baez y Córdova (2018), "los sistemas ERP (Enterprise Resource Planning) se han definido como un sistema global de planificación de los recursos y de gestión de la información que de forma estructurada pueden satisfacer la demanda de las necesidades de gestión de la empresa" (p. 2).

Por su parte, Oltra (2012) señala que "en su continua evolución, han pasado de ser una mera herramienta de trabajo, a ser un elemento competitivo y estratégico, llegando incluso a generar nuevos modelos de negocio basados en su desarrollo" (p. 5).

\section{METODOLOGÍA}

El tipo de investigación realizada es de tipo aplicada y descriptiva. Aplicada porque se determinará y comprobará el beneficio de la implementación del sistema ERP en una empresa del sector salud, y descriptiva porque se mencionarán los atributos de la implementación del sistema ERP en las cuentas por cobrar del módulo de caja.

Según Lozada (2014), la investigación aplicada tiene por objetivo la generación de conocimiento con aplicación directa y a mediano plazo en la sociedad o en el sector productivo.

Para Hernández, Fernández y Baptista (2010), "con frecuencia, la meta del investigador consiste en describir fenómenos, situaciones, contextos y eventos; esto es, detallar como son y se manifiestan. Los estudios descriptivos buscan especificar las propiedades, las características y los perfiles de personas, grupos, comunidades, procesos, objetos o cualquier otro fenómeno que se someta a un análisis" (p. 80).

En cuanto al diseño de investigación, esta es no experimental en virtud de que la variable, objeto de estudio, no será manipulada.
Se aplicaron instrumentos de recolección de datos como entrevistas y encuestas a los usuarios que utilizan el sistema Spring ERP/módulo de caja.

En el caso de las entrevistas, se aplicaron mediciones y comparaciones de rendimiento a los sistemas Spring ERP y el sistema Msante para determinar la cantidad de pacientes que pueden ser atendidos por usuario en cada caso y así determinar la mejora con el sistema ERP.

Para el caso de las encuestas, se comprobaron, corroboraron y demostraron los beneficios de la implementación del sistema Spring ERP/Modulo de caja.

\section{RESULTADOS}

Los siguientes cuadros y figuras representan los resultados obtenidos del trabajo de campo de la variable independiente, aplicado al universo de quince usuarios del módulo de caja, mediante entrevistas y encuestas.

Se ha empleado como unidad de medida el tiempo en minutos que requieren tanto el sistema Spring ERP como el sistema Msante para atender un paciente.

Asimismo, para determinar la cantidad de atenciones a pacientes que soporta cada sistema, se promediaron las respuestas obtenidas de los usuarios entrevistados. Por ejemplo, en la pregunta 1 de la entrevista, se enunció lo siguiente: “ ¿El sistema Spring ERP ha dado mejoras a la parte operativa, reduciendo o eliminando el trabajo manual? ¿Cuánto tiempo aplicaba por paciente en cada sistema?"

Así, se determinó el tiempo que se necesitaba por paciente en cada sistema, lo cual se refleja en la Tabla 1.

Se debe considerar que una hora equivale a $60 \mathrm{mi}-$ nutos, entonces:

- Spring $=60 / 3.75=16$ pacientes por hora

- Msante $=60 / 22.50=2.66$ pacientes por hora

También se incluyen las horas hombres empleadas durante la semana, que son 12 horas de trabajo de lunes a sábado. No se consideraron los domingos, dado que ese día no es laborable.

Aplicando el siguiente cálculo se obtiene: 
- Total de horas invertidas por cajero/usuario en un día $=12$ horas

- Total de días laborables $=26$ días

- Total de horas trabajadas en un mes (26 días $\mathrm{x}$ 12 horas) $=312$ horas

En la Tabla 2 se detalla el grado de eficiencia según el tiempo que tarda un usuario en ejecutar cada sistema al atender un paciente; primero, los minutos que se tarda por paciente, luego, la cantidad de pacientes atendidos por hora y por mes.
El cálculo por mes se obtiene del siguiente procedimiento:

- Spring $=16$ pacientes $\times 312$ horas $=4992$ pacientes.

- Msante $=2.66$ pacientes $\times 312$ horas $=830$ pacientes.

En la Figura 1 se aprecia el resultado de atenciones al mes por usuario y por sistema. El actual módulo de caja Spring ERP muestra un mejoramiento en la labor por cajero, ya que incrementa su rendimiento

Tabla 1. Tiempo utilizado en cada sistema por usuario en referencia a la atención por paciente.

\begin{tabular}{|l|c|c|}
\hline \multicolumn{1}{|c|}{ Usuario } & Sistema Spring ERP & Sistema Msante \\
\hline Usuario 1 & 4 minutos & 10 minutos \\
\hline Usuario 2 & 3 minutos & 30 minutos \\
\hline Usuario 3 & 3 minutos & 20 minutos \\
\hline Usuario 4 & 5 minutos & 30 minutos \\
\hline Suma de tiempo empleado & 15 minutos & 100 minutos \\
\hline Promedio de tiempo empleado por paciente & 3.75 minutos & 22.50 minutos \\
\hline
\end{tabular}

Fuente: Elaboración propia.

Tabla 2. Análisis de eficiencia de atención de pacientes por cada sistema.

\begin{tabular}{|l|c|c|c|}
\hline \multicolumn{1}{|c|}{ Sistema } & Minutos/paciente & Paciente/hora & Paciente/mes \\
\hline Spring ERP & 3.75 & 16.00 & 4992 \\
\hline Msante & 22.50 & 2.66 & 830 \\
\hline
\end{tabular}

Fuente. Elaboración propia.

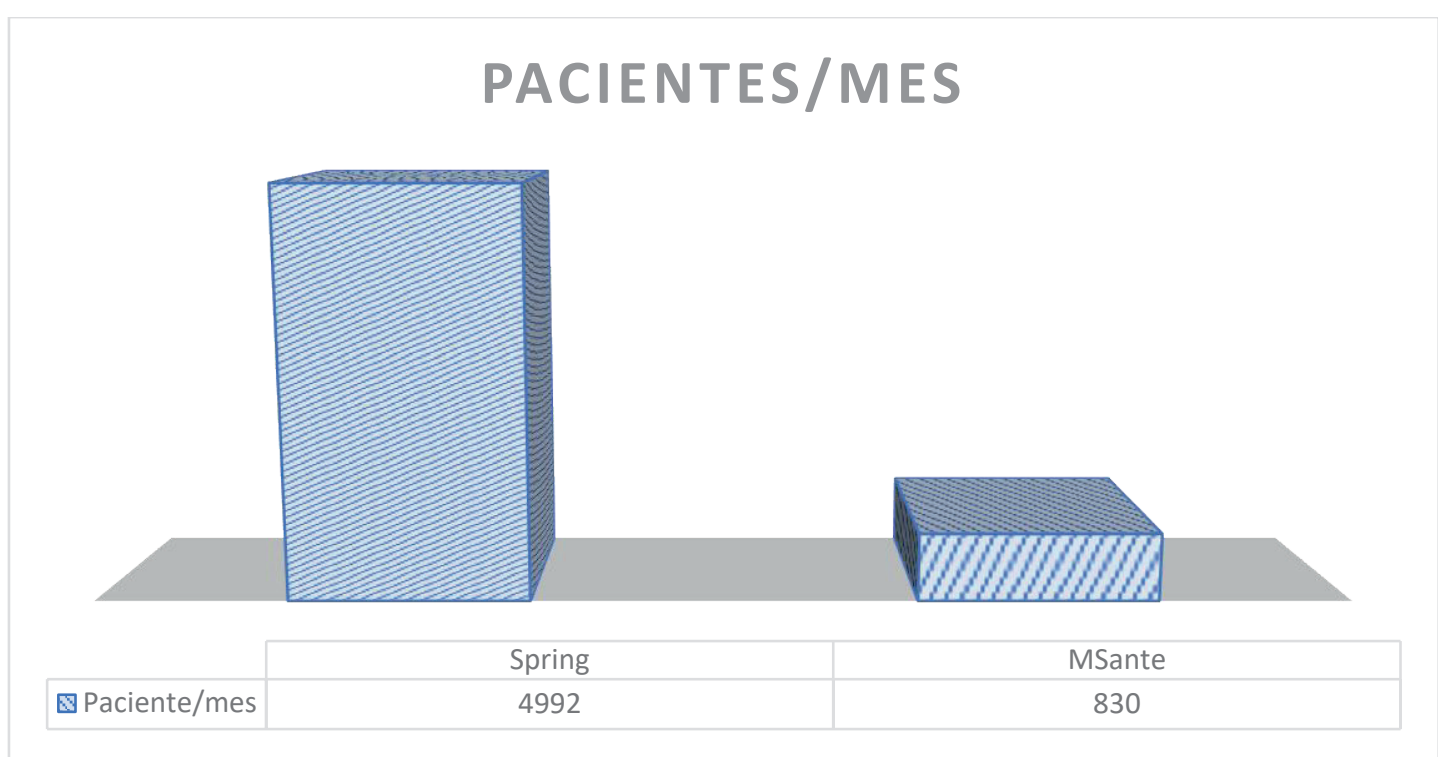

Figura 1. Análisis de rendimiento de cada sistema con respecto a la cantidad de atención de pacientes por mes.

Fuente: Elaboración propia. 
IMPLEMENTACIÓN DE UN SISTEMA INTEGRADO DE PLANIFICACIÓN DE RECURSOS EMPRESARIALES PARA MEJORAR LA PRODUCTIVIDAD EN LAS RECAUDACIONES POR CAJA DE UNA IMPORTANTE CLIIIICA DE LA CIUDAD DE LIMA

mensual en más de 4000 pacientes, mientras que el sistema Msante tan solo permitía una atención por usuario de hasta 830 pacientes.

Al comparar, se determina un incremento porcentual en $501.45 \%$.

En la Tabla 3, según las entrevistas realizadas, se midió la eficacia basada en el tiempo que un usuario empleaba en la labor de cobranza por caja en cada sistema, examinando la capacidad de cobro por paciente en minutos, horas y mes.

En la Figura 2, se puede apreciar el rendimiento en atención a pacientes que puede llegar a ofrecer cada sistema, por cajero, en la labor de cobranzas. El sistema ERP mejora la capacidad de atención mensual al atender hasta 6808 pacientes, mientras que el sistema anterior soportaba hasta 2203 pacientes, lo cual se traduce en un incremento porcentual de $209.03 \%$, tal como se detalla a continuación:

Asimismo, al consultar de qué manera contribuía este sistema ERP en el tema del control, se obtuvieron los siguientes resultados de parte de los usuarios más experimentados, los cuales establecieron nueve bondades de este sistema:

Se analizó, además, la variable dependiente para medir la productividad de cada programa informático, seleccionando a cuatro usuarios del módulo de caja que conocían ambos sistemas y que permanecieron en la clínica luego de la activación del nuevo sistema.

Según Argota y Argota (2018) "La prueba $t$-Student es una de las más utilizadas para la comparación de dos muestras en diferentes escenarios de interés" (p. 2).

Se determinaron los escenarios en base a la hipótesis general "La implementación de un sistema ERP mejora el control de las recaudaciones por caja en una importante clínica ubicada en la ciudad de Lima".

Para probar la hipótesis, se realizó la prueba $t$-Student; se examinaron los minutos de atención por paciente antes y después de la implementación, tal como se aprecia en la Tabla 4:

Tabla 3. Análisis de eficacia en la labor de las cobranzas por caja.

\begin{tabular}{|c|c|c|c|}
\hline Sistema & Minutos/paciente & Paciente/hora & Paciente/mes \\
\hline Spring & 2.75 & 21.82 & 6808 \\
\hline Msante & 8.50 & 7.06 & 2203 \\
\hline
\end{tabular}

Fuente. Elaboración propia.

\section{PACIENTE/MES}

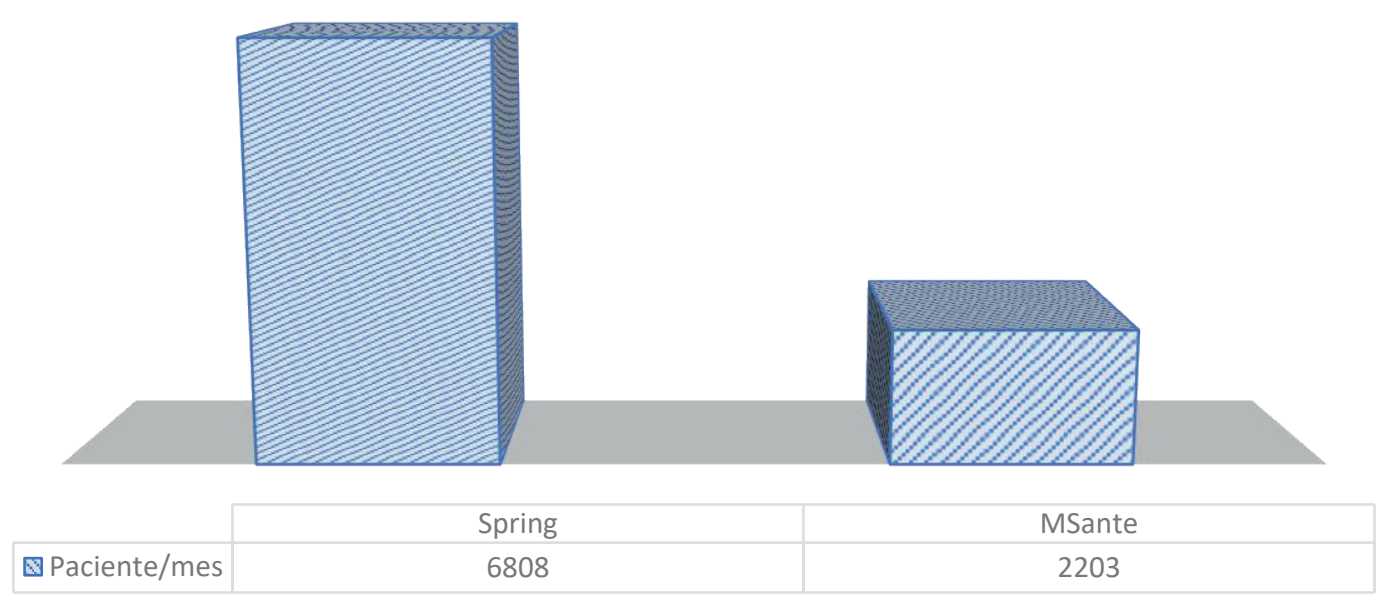

Figura 2. Análisis de rendimiento en atención a pacientes que ofrece cada sistema por cajero en la labor de cobranzas por caja.

Fuente. Elaboración propia. 


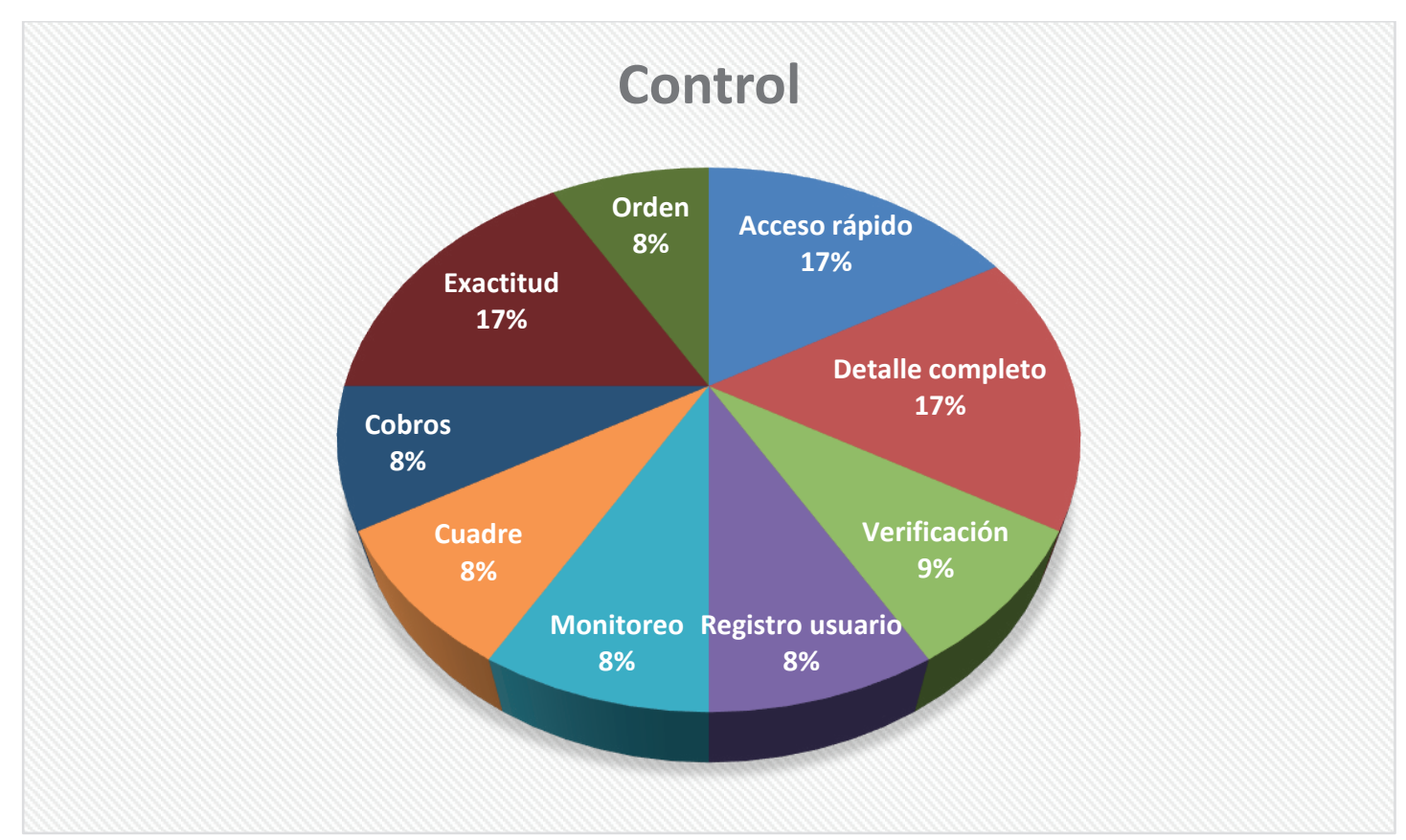

Figura 3. Análisis de factores de control. Fuente: Elaboración propia.

Tabla 4. Análisis de muestras en minutos de atención por paciente.

\begin{tabular}{|c|c|c|c|}
\hline Usuario & Preimplementación & Posimplementación & $\overline{\mathrm{d}}$ \\
\hline 1 & 10 & 4 & 6 \\
\hline 2 & 30 & 3 & 27 \\
\hline 3 & 20 & 3 & 25 \\
\hline 4 & 30 & 5 & 17 \\
\hline
\end{tabular}

Fuente. Elaboración propia.

Donde:

$\mathrm{H}_{0}=\mu \mathrm{d} \leq 0$

$\mathrm{H}_{1}=\mu \mathrm{d}>0$

Al denotar tenemos:

$\mathrm{H}_{0}=$ No mejora el control de las recaudaciones por caja.

$\mathrm{H}_{1}=$ Mejora el control de las recaudaciones por caja.

Además:

Si $p$-valor $<\alpha \rightarrow$ Se rechaza $\mathrm{H}_{0}$.

Si $p$-valor $\geq \alpha \rightarrow$ Se acepta $\mathrm{H}_{0}$.

Con base en lo mostrado en la Tabla 4, se obtienen los siguientes resultados del análisis de la prueba de hipótesis:
Cálculo de la media: $\quad \overline{\mathrm{d}}=18.75$

Usuarios:

$\mathrm{n}=4$

Grado de libertad:

$\mathrm{gl}=(\mathrm{n}-1)=3$

Desviación estándar: $\mathrm{Sd}=\sqrt{(d 1-d)^{\wedge} 2 / n-1}=$ 9.53502316

Estadístico de prueba: $\quad \mathrm{t}=\frac{\overline{\mathrm{d}}}{\mathrm{Sd} / \sqrt{\mathrm{n}}}=3.93286931$

Valor crítico:

$t(1-\alpha)(n-1)=2.35336343$

Nivel de significancia: $\quad \alpha=0.05 \rightarrow 5 \%$

p-valor

0.01463716

Decisión:No se debe utilizar la hipótesis nula.

Luego de analizar la prueba de hipótesis, se verificó que el estadístico de prueba es mayor al valor crítico y que el resultado de $p$-valor es menor al valor $\alpha$; 
por consiguiente, se rechaza la hipótesis nula $(\mathrm{HO})$ y se acepta la hipótesis alternativa (H1). Además, se observó que la media es de 18.75, por lo que la asimetría es hacia la derecha.

Asimismo, se tomaron otros tipos de análisis para la demostración de las hipótesis, en los que se corroboraron las mejoras posimplementación mediante las encuestas realizadas a los usuarios del sistema ERP/Módulo de caja, quienes dieron repuestas favorables con respecto a la implementación.

No obstante, se establecieron escalas de medición para llegar a saber el grado de aceptabilidad; los resultados se muestran en la Tabla 5:

La prueba de la hipótesis general se basó en encuestas aplicadas a 11 usuarios del módulo de caja.
Esta prueba obtuvo un resultado de 49 puntos en promedio de un total de 55 , que representa un $89 \%$, lo que demostró que la implementación del sistema ERP en la clínica tiene aceptabilidad, como se refleja en la Figura 4:

Adicionalmente, para determinar su grado de aceptación, se contrastaron las tres hipótesis específicas con sus respectivos indicadores.

La primera hipótesis específica elaborada fue "La automatización de operaciones por medio del sistema Spring ERP/módulo de caja contribuirá con el control de las recaudaciones por caja en una importante clínica ubicada en la ciudad de Lima".

Se establecieron ocho indicadores; la medida de aceptabilidad de cada uno superó el $80 \%$, lo que

Tabla 5. Puntajes de medición y escala de valor.

\begin{tabular}{|l|c|c|}
\hline \multicolumn{1}{|c|}{ Determinación } & Puntaje & Grado porcentual de valor \\
\hline Excelente & 5 & $81-100 \%$ \\
\hline Muy Bueno & 4 & $61-80 \%$ \\
\hline Bueno & 3 & $41-60 \%$ \\
\hline Regular & 2 & $21-40 \%$ \\
\hline Deficiente & 1 & $0-20 \%$ \\
\hline
\end{tabular}

Fuente: Elaboración propia.

GRADO PORCENTUAL DE VALOR DE ACEPTACIÓN

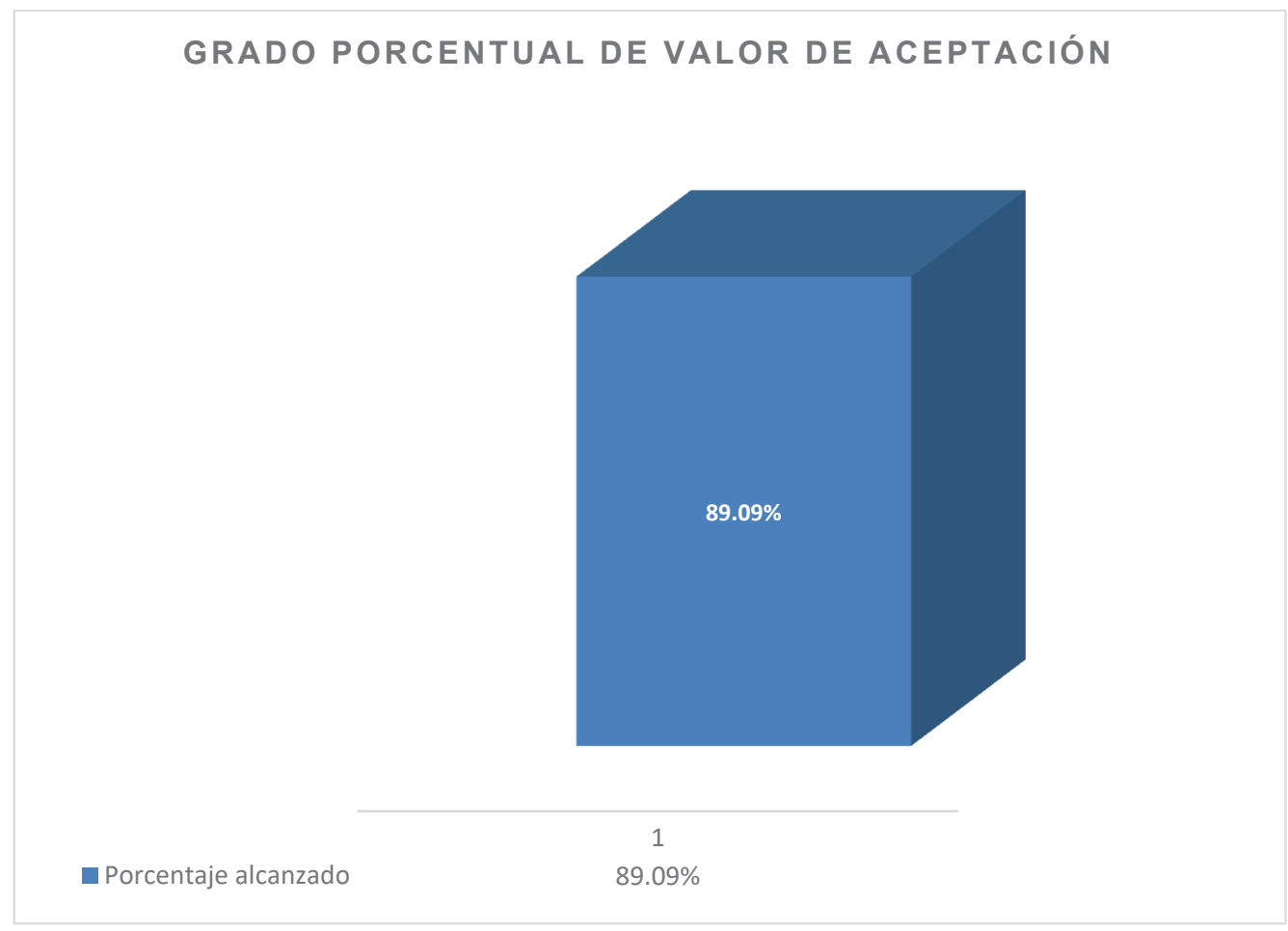

Figura 4. Aceptabilidad de la hipótesis general.

Fuente: Elaboración propia. 
resultó en que la primera hipótesis específica fuera aceptada, tal como se muestra en la Figura 5.

La segunda hipótesis específica elaborada fue "La mejora del proceso de información por medio del sistema Spring ERP/módulo de caja acrecentará el control de las recaudaciones por caja en una importante clínica ubicada en la ciudad de Lima".

Se establecieron seis indicadores; la medida de aceptabilidad de cada uno superó el $80 \%$, lo que resultó en que la segunda hipótesis específica fuera aceptada, tal como se muestra en la Figura 6.

La tercera hipótesis específica elaborada fue "Los arqueos de caja por medio del sistema Spring ERP/ módulo de caja generarán un adecuado control de las recaudaciones por caja en una importante clínica ubicada en la ciudad de Lima".
Se establecieron diez indicadores; la medida de aceptabilidad de cada uno superó el $80 \%$, lo que resultó en que la tercera hipótesis específica fuera aceptada, tal como se muestra en la Figura 7:

\section{DISCUSIÓN DE RESULTADOS}

Una vez expuestos los resultados de este trabajo de investigación, obtenidos del trabajo de campo mediante encuestas y entrevistas, se corrobora que la implementación de un sistema ERP para el mejoramiento del control ha sido exitosa, lo que se refleja en el grado de aceptabilidad.

Indudablemente, el uso del sistema ERP ha resultado en cambios significativos en la clínica, puesto que se observaron mejoras en la maximización de la productividad y una mejor plataforma de soporte y atención.

\section{GRADO PORCENTUAL DE ACEPTACIÓN}

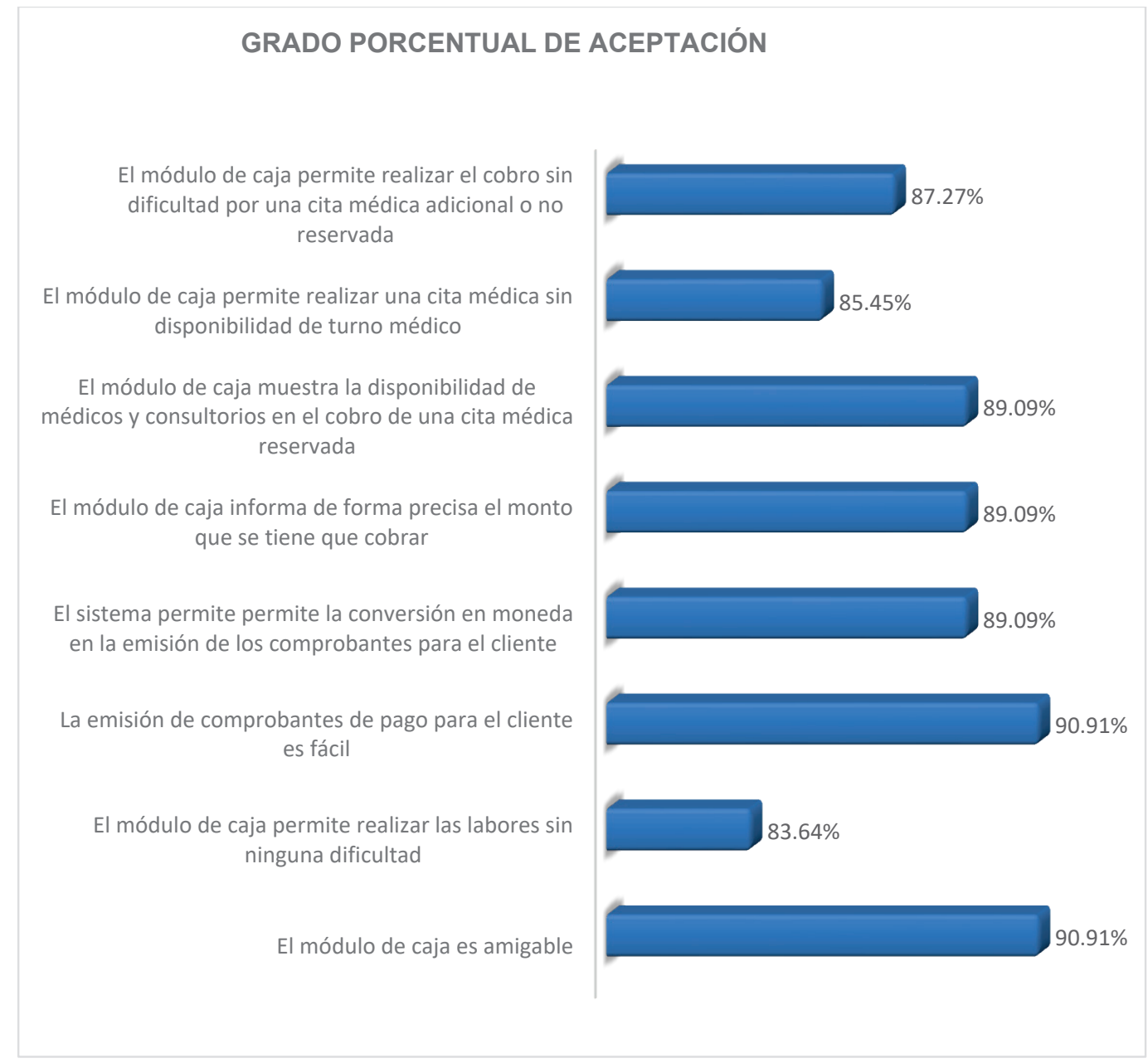

Figura 5. Indicadores de aceptabilidad de la primera hipótesis específica.

Fuente: Elaboración propia. 
IMPLEMENTACIÓN DE UN SISTEMA INTEGRADO DE PLANIFICACIÓN DE RECURSOS EMPRESARIALES PARA MEJORAR LA PRODUCTIVIDAD EN LAS RECAUDACIONES POR CAJA DE UNA IMPORTANTE CLIIIICA DE LA CIUDAD DE LIMA

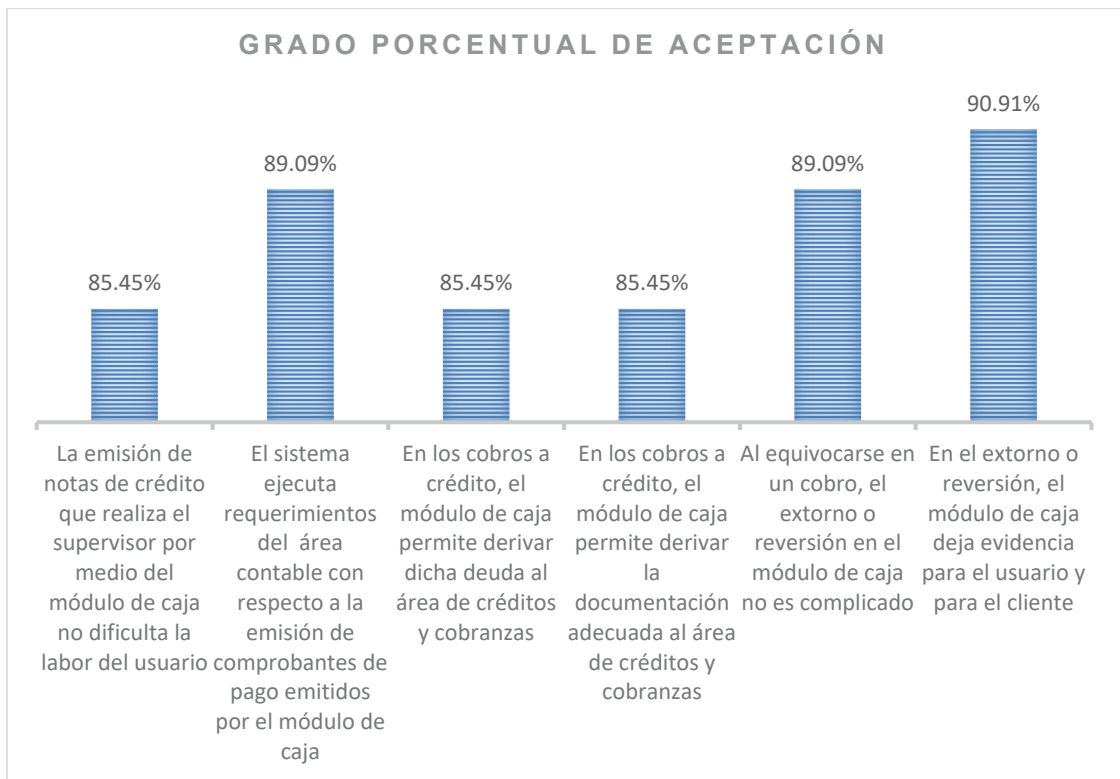

Figura 6. Indicadores de aceptabilidad de la segunda hipótesis específica.

Fuente: Elaboración propia.

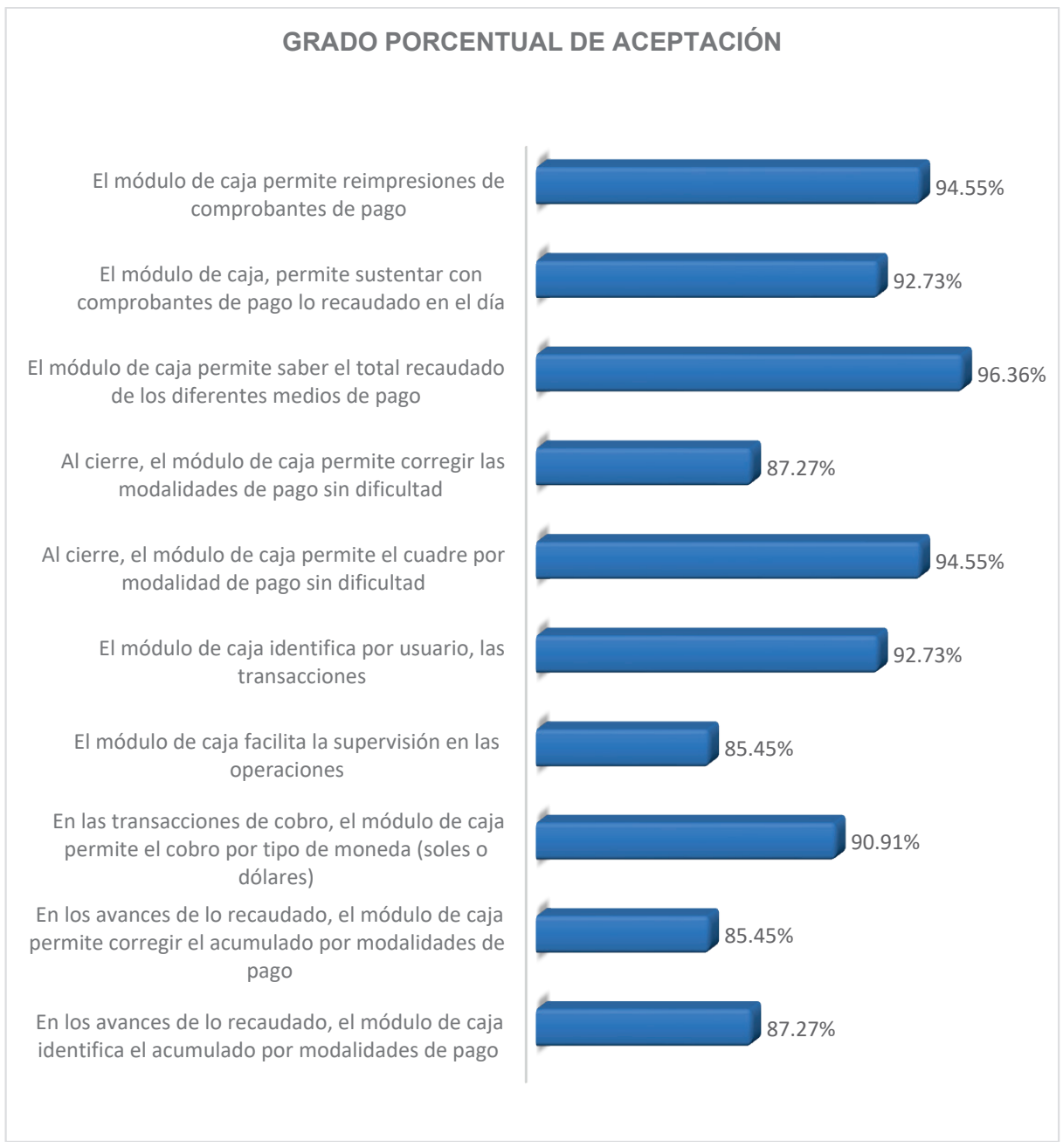

Figura 7. Indicadores de aceptabilidad de la tercera hipótesis específica. Fuente. Elaboración propia. 
La automatización, el mejoramiento u ordenamiento de los procesos de información y los diversos reportes de caja para un adecuado arqueo mejoraron el control y han sido recibidos agradablemente por los usuarios de caja, en virtud de que han demostrado que los tiempos de atención se minimizaron y ellos logran culminar sus labores en la hora establecida.

\section{CONCLUSIONES}

La implementación de un sistema ERP en la clínica ha dado los resultados esperados, tal como se ha demostrado, puesto que mejora aspectos no solo de control de las recaudaciones por caja, sino también de tiempos de atención por paciente, actividades y procesos, por lo que es un sistema adaptable al entorno.

La implementación del sistema ERP en la clínica ha permitido descubrir que este sistema ofrece una plataforma de soporte o capacidad para el máximo aprovechamiento de los recursos, ya que se logra optimizar el tiempo y agilizar la operatividad de los usuarios.

La automatización de actividades por medio del Spring ERP/módulo de caja ha demostrado que es amigable para los usuarios, les permite realizar sus labores sin dificultad, facilita la emisión de comprobantes de pago y la conversión de monedas y contribuye en la precisión de los cobros; asimismo, proporciona información sobre la disponibilidad de médicos y consultorios y posibilita la reserva y cobro de citas médicas no reservadas a tiempo.

El mejoramiento del proceso de información por medio del sistema Spring ERP/Módulo de caja permite que la emisión de las notas de crédito hechas por los supervisores no dificulte la labor de los usuarios; ha logrado que no se tengan incidencias con el área contable; hace más fácil el traslado de la documentación de una deuda, o cobro a crédito, al área de créditos y cobranzas; y deja evidencia de los extornos de las operaciones.

Los arqueos de caja mediante el sistema Spring ERP/módulo de caja permiten un mejor funcionamiento en los avances de caja ya que permite identificar lo recaudado según el medio de pago; permite la corrección de los medios de pago sin dificultad; facilita las transacciones de cobro por tipo de moneda; facilita la supervisión de caja; y permite que, al cierre caja, se genere un reporte de cuadre y presentación. Asimismo, este sistema ha contribuido en un mejor cuadre de caja, puesto que permite adecuar y corregir las modalidades de pago. Con respecto a la emisión del reporte de cuadre, el sis- tema permite saber el total recaudado por medios de pago, sustentar adecuadamente cada operación e imprimir comprobantes obviados.

\section{REFERENCIAS BIBLIOGRÁFICAS}

[1] Argota, G., y Argota H. (2018). Aplicación de la prueba t-Student para la competencia técnica y trazabilidad analítica: ejemplo de estudio. Campus, 23 (26), 145-150. Recuperado de https://doi.org/10.24265/campus.2018. v23n26.04

[2] Duke, V., Navarro, M., Díaz, G., Pérez, Y., y Vargas-Lombardo, M. (2016). Exploración en los sistemas CRM/ERP como estrategia en el sector PYMES. Revista de Iniciación Científica, 2(2), 86-94. Recuperado de https://revistas. utp.ac.pa/index.php/ric/article/view/1251/1414

[3] ESAN. (14 de setiembre de 2018). El sistema ERP en la gestión de proyectos. Recuperado de https://www.esan.edu.pe/apuntesempresariales/2018/09/el-sistema-erp-en-lagestion-de-proyectos/

[4] Farfán, J. (2020). La implementación de un sistema automatizado reduce los tiempos de atención en los procesos aplicables a la ventanilla única de turismo en la Municipalidad Provincial del Callao. Industrial Data, 23(2), 3137. Recuperado de http://dx.doi.org/10.15381/ idata.v23i2.15566

[5] Fernández, A. (2015). Gestión y Control administrativo de las operaciones de caja. Madrid, España: Editorial Elearning S.L.

[6] Guerrero, A., Marín, M., y Bonilla, D. (2018). Erp como alternativa de eficiencia en la gestión financiera de las empresas. Revista Lasallista de Investigación, 15(2), 182-193. Recuperado de https://doi.org/10.22507/rli.v15n2a14

[7] Hernández, I., Báez, P., y Córdova, J. (2018). Desarrollo de software de simulación ERP para herramienta didáctica en el proceso de enseñanza-aprendizaje. Revista de Tecnologías de la Información, 5(17), 1-8. Recuperado de https://www. ecorfan.org/bolivia/researchjournals/ Tecnologias_de_la_Informacion/vol5num17/ Revista_de_Tecnolog\%c3\%adas_de_la_ Informaci\%c3\%b3n_V5_N17_1.pdf

[8] Hernández, R., Fernández, C., y Baptista, L. (2010). Metodología de La Investigación. México D.F, México: McGraw-Hill/ Interamericana Editores S.A de CV. 
[9] Hernández, J., Carreño, M., Sandoval, J., Estrada, I., y Ignacio, R., (2016). Propuesta de criterios de evaluación en la implementación de ERP's en instituciones de educación superior. Pistas Educativas, 38(122), 232243. Recuperado de http://itcelaya.edu.mx/ojs/ index.php/pistas/article/view/679

[10] Hurtado, E., Arroyo, N., y Guzmán, F. (2019). El Control interno y la importancia de su aplicación en las compañías. Revista Observatorio de la Economía Latinoamericana, (agosto 2019). Recuperado de https://www.eumed.net/rev/ oel/2019/08/control-interno-companias.html

[11] Lozada, J. (2014). Investigación aplicada: Definición, propiedad intelectual e industria. CienciAmérica 3(1), 34-39. Recuperado de http://cienciamerica.uti.edu.ec/openjournal/ index.php/uti/article/view/30

[12] Mayorga, M., Espinosa, M., López, A., y Chango, M. (2020). Control interno para el área de cajas en Cooperativas de Ahorro y Crédito. Caso de estudio: Cooperativa de Ahorro y
Crédito Indígenas Galápagos Ltda. Visionario Digital, 4(2), 57-80. Recuperado de https://doi. org/10.33262/visionariodigital.v4i2.1215

[13] Núñez, R. (2016). Software ERP. Análisis y Consultoría de Software Empresarial. Madrid, España: IT Campus Academy.

[14] Oltra Badenes, R. (2012). Sistemas Integrados de Gestión Empresarial. Evolución histórica y tendencias de futuro. Valencia, España: Universitat Politècnica de València.

[15] Riascos, S., y Arias, V. (2016). Análisis del impacto organizacional en el proceso de implementación de los Sistemas de Información ERP - Caso de estudio. Entramado, 12(1), 284302. Recuperado de http://dx.doi.org/10.18041/ entramado.2016v12n1.23127

[16] Suárez, C. (2016). Guía Práctica. Software de gestión empresarial. Madrid, España: Edisa. 


\section{Implementation of an Enterprise Resource Planning System to Improve the Productivity of Cash Register Collections of an Important Clinic in the City of Lima}

JUAN FAUSTINO INFANTES LoO ${ }^{1}$ Santiago Domingo Moquillaza Henríquez ${ }^{2}$

\begin{abstract}
The purpose of this study is to show the results obtained after the implementation of an enterprise resource planning system in an important clinic in the city of Lima. Quantitative and qualitative techniques were applied to determine the improvements or benefits that arose from implementation. The results are shown in tables and graphs that were prepared based on the information obtained from the users of the ERP system/cash register module. It is concluded that a better control of cash register collections contributes to better productivity performance and better customer service.
\end{abstract}

Keywords: ERP system; Msante system; automation; SUNAT; control.

\section{INTRODUCTION}

Companies need to be aligned with information systems to achieve innovation, competitivity and a better position in the market. This is possible through the application of agile processes and activities that enable operational efficiency and effectiveness, which result in the acquisition of optimal information for good decision-making and continuous improvement.

This study presents the knowledge acquisition of the enterprise resource planning (ERP) systems implemented at a clinic in the city of Lima. This study also shows the way in which these systems contribute (1) to the work executed by each employee or user, since ERP is an easy and comfortable work tool for good performance of the tasks assigned, and (2) to production improvements of the clinic through information integration.

When observing and analyzing the enterprise resource planning system implemented at the clinic, it becomes evident that it enables planning each activity in an orderly manner while respecting established procedures; improves the flow of documents; and optimizes time to comply with the requirements of supervisorial bodies, such as SUNAT (Peruvian National Superintendency of Tax Administration). In this way, control of the cash process is achieved, which has an impact on the results of money collections.

ERP systems were created for companies that handle manufacturing or stock inventories; however, the novelty of this study resides in the fact that it shows that this system can also be applied to a service company. In the same way, this study will make it possible to expand knowledge of the ERP system by proposing its applicability in a company in the health services sector, which is demonstrated by processing the information provided by the users of the clinic's cash register module on the benefits from the ERP system. professor at Instituto Certus (Lima, Peru)

ORCID: https://orcid.org/0000-0002-5384-718X

Corresponding author: jinfantes1@certus.edu.pe

2 Degree in Computer Science from UNMSM (Lima, Peru). Currently working as professor at UNMSM (Lima, Perú)

ORCID: https://orcid.org/0000-0001-9531-881X

E-mail: smoquillazah@unmsm.edu.pe 
According to Duke, Navarro, Díaz, Pérez, and Vargas-Lombardo (2016), "los principales retos que presentan las organizaciones hoy, es enfrentar los constantes cambios al mercado actual, con la necesidad de implementar sistemas que ayuden a la buena comunicación e integración entre las distintas áreas de una organización [the main challenges faced by the organizations these days is to face the continuous changes in the current market and the need to implement systems that help good communication and integration among the different areas of an organization]" (p. 87).

Therefore, the need arises to integrally organize activities and processes, since it is necessary for each department, division or area of every business to always maintain fluid and accurate communication. At the same time, each operational area must interact with each unit of the organization to search for improved control that enables decision-making based on correct information.

Undoubtedly, the proper functioning of the ERP system will depend on an adequate configuration, the predisposition of the personnel, as well as their experience. Given the investment cost that this type of system requires, there is no doubt that benefits will be obtained in the medium and long term. Therefore, improvements must be made on the proper processing or transfer of information so that the relevant or specialized area has the correct data; otherwise, the information provided will not be accurate.

In view of the above, the general objective was "to implement an ERP system to improve the control of cash register collections in an important clinic located in the city of Lima". Likewise, the specific objectives were "to demonstrate that the automation of operations through the Spring ERP system/ cash register module will contribute to the control of cash register collections in an important clinic located in the city of Lima", "to corroborate that the improvement of the information process by means of the Spring ERP system/cash register module will increase the control of the cash register collections of an important clinic located in the city of Lima" and "to prove that cash counts by means of the Spring ERP system/cash register module will generate an adequate control of the cash register collections of an important clinic located in the city of Lima".

An advantage of the ERP system is that it contributes to the fulfilment of objectives through parameterization.

As defined by Suárez (2016):
La parametrización de un ERP permite adaptar el funcionamiento del sistema a las necesidades concretas de cada empresa, así como incorporar nuevas funciones $o$ modos de funcionamiento a medida que la empresa en cuestión lo requiere, sin requerir de desarrollos específicos o a medida del cliente [The parameterization of an ERP allows to adjust the functioning of the system to the specific needs of each company and also to incorporate new functions or modes of operation as the company in question requires it, without requiring specific or client-customized developments]. (p. 25)

\section{GENERAL HYPOTHESIS}

The implementation of an ERP system improves the control of the cash register collections in an important clinic located in the city of Lima.

\section{SPECIFIC HYPOTHESES}

1. The automation of operations by means of the Spring ERP system/cash register module will contribute to the control of cash register collections in an important clinic located in the city of Lima.

2. The improvement of the information process by means of the Spring ERP system/cash collection module will increase the control of cash register collections in an important clinic located in the city of Lima.

3. The cash counts by means of the Spring ERP system/cash module will generate an adequate control of cash register collections in an important clinic located in the city of Lima.

\section{JUSTIFICATION}

According to Hernández, Carreño, Sandoval, Estrada and Ignacio (2016), "desde hace más de tres décadas los Sistemas Enterprise Resource Planning (ERP) en inglés, conocidos como Sistemas de Planificación de Recursos, Empresariales (sic), han otorgado alta competitividad al mundo empresarial [for more than three decades, enterprise resource planning (ERP) systems have made the business world highly competitive]" (p. 234). Based on this, the implementation of an ERP system in an important clinic located in the city of Lima became necessary to improve the processes that contribute to the control of cash collections or cashing through the cash register module. Likewise, this implementation 
would contribute to the improvement of customer or patient care and information procedures and would eliminate manual procedures to facilitate the cashier's work, provide correct information and optimize accounting department activities so that it handles correct financial information.

In words of Farfán (2020), "a través de la automatización de la gestión por procesos, las organizaciones pueden tener un mejor funcionamiento, aumentando la eficiencia de las mismas, lo cual representa una ventaja competitiva [through the automation of process management, organizations function better and their efficiency increase, which represent a competitive advantage]" (p. 36).

The information system makes it possible to design better user-friendly IT structures for a good review and supervision of operations, which allows the accounting department to verify each operation and to ensure compliance with the rules of the supervisory and control bodies.

\section{According to Fernández (2015):}

El arqueo de caja "consiste en la realización de una [sic] análisis de la [sic] transacciones de efectivo que se han producido en al [sic] empresa en un lapso de tiempo determinado". La finalidad del mismo es comprobar si existen diferencias entre el efectivo contabilizado y el recuento de billetes, monedas y cheques físicamente que tenemos en nuestra caja. Hemos de tener en cuenta que todo registro contable debe estar soportado documentalmente, de modo que cualquier error u omisión debe ser fácilmente detectable [The cash count "consists of carrying out an analysis of the cash transactions that have taken place in the company over a given period of time". Its purpose is to check whether there are any differences between the cash accounted for and the physical count of notes, coins and cheques in our cash register. It should be borne in mind that all accounting records must be supported by documentation, so that any errors or omissions should be easily detectable]. (p. 94)

During the cash count documentation is checked and controlled, which makes it possible to accurately account for the different payment methods. The cash count is included in an ERP system in order to comply with the banking and the payment of the tax on financial transactions (ITF), as stipulated by Law 28194, in force since 2004.
Mayorga, Espinosa, López and Chango (2020) state that "los sistemas de información permiten a la organización captar, identificar y comunicar de forma adecuada el plazo permitido al personal para afrontar sus responsabilidades. La comunicación debe ser fluida en todo el conjunto de la institución [information systems allow the organization to capture, identify and adequately communicate the information related to the time allowed to staff to meet their responsibilities. Communication must be fluid throughout the institution]" (p. 62).

According to Hurtado, Arroyo and Guzmán (2019), "el control interno puede ayudar a que una entidad consiga sus objetivos de rentabilidad y rendimiento y a prevenir la pérdida de recursos [internal control can help an entity to achieve its profitability and performance objectives and prevent the loss of resources]" (p. 6).

The implementation of the ERP system has managed to keep an important private clinic in the city of Lima - which has a history of more than 150 years and has three locations in the districts of Lima, Chorrillos and Surco- in a leading position.

\section{ENTERPRISE RESOURCE PLANNING SYSTEMS}

According to Riascos and Arias (2016), "la decisión de implementar un sistema de información ERP le facilita a la organización desarrollar sus procesos administrativos de forma eficiente $y$ eficaz [the decision to implement an ERP information system makes it easier for the organization to develop its administrative processes efficiently and effectively]" (p. 289).

The ERP system is an adaptable program that provides optimal management and operational results, since it makes it possible to make the most of the resources the system provides in an integrated manner. This system allows to configure or shape the various tasks in a structured manner according to the user's profile; in this way, it provides reliable information that is backed up by a database platform.

According to Núñez (2016):

Típicamente, un sistema ERP es un sistema de información integrado en la forma de un paquete de software compuesto por varios módulos, tales como producción, ventas, finanzas y recursos humanos, que nos aporta una integración de datos horizontales a lo largo de la organización y a través de sus procesos de negocio. Esos paquetes pueden ser personalizados de forma que 
respondan a las necesidades específicas de la organización [Typically, an ERP system is an integrated information system in the form of a software package that consists of several modules, such as production, sales, finance and human resources, which provides horizontal data integration throughout the organization and across its business processes. These packages can be customized to meet the specific needs of the organization]. (p. 7)

Moreover, Oliva, Ortigoza and Méndez point out "Los sistemas ERP son soluciones de software de gestión de información que se ocupa de las necesidades de las empresas de manera integral a través de la automatización de procesos operativos [ERP systems are information management software solutions that address the needs of companies in a comprehensive manner through the automation of operational processes]" (2012, p. 2, as cited in Guerrero, Marín \& Bonilla, 2018, p. 185).

According to Suárez (2016), "podemos definir un sistema ERP como un software integrado de gestión empresarial, compuesto por un conjunto de módulos funcionales (logística, finanzas, recursos humanos, etc.) susceptibles de ser adaptados a las necesidades de cada cliente [we can define an ERP system as an integrated business management software that consists of a set of functional modules (logistics, finance, human resources, etc.) that can be adapted to the needs of each client]" (p. 19).

Automation of an ERP system is achieved when activities are carried out in a simple manner in the shortest possible time; however, unless the activities and processes are clear, the results obtained will remain unchanged.

It is essential to consider the accounting area within an ERP system since it is an area where information is centralized. Thus, accounting participation is essential in each area or business unit as it contributes to the improvement of operational designs or structures; it facilitates the flow of accounting information and makes it reliable.

According to information published in ESAN (September 14, 2018), "el sistema de planificación de recursos empresariales o enterprise resource planning $(E R P)$ es un software desarrollado para gestionar los principales procesos empresariales [the enterprise resource planning (ERP) system is a software developed to manage the main business processes]".
According to Hernández, Baez and Córdova (2018), "los sistemas ERP (Enterprise Resource Planning) se han definido como un sistema global de planificación de los recursos y de gestión de la información que de forma estructurada pueden satisfacer la demanda de las necesidades de gestión de la empresa [ERP (Enterprise Resource Planning) systems have been defined as global systems of resource planning and information management that can meet the demand of the company's management needs in a structured manner]" (p. 2).

Oltra (2012) states that "en su continua evolución, han pasado de ser una mera herramienta de trabajo, a ser un elemento competitivo y estratégico, llegando incluso a generar nuevos modelos de negocio basados en su desarrollo [in their continuous evolution, [ERPs] have gone from being a simple work tool to being a competitive and strategic element that even generates new business models based on their development]" (p. 5).

\section{METHODOLOGY}

This research is applied and descriptive. It is applied because it will determine and verify the benefits of the implementation of the ERP system in a company in the health sector; and it is descriptive because it mentions the attributes of the ERP system implementation in the accounts receivable of the cash register module.

According to Lozada (2014), applied research aims to generate knowledge with direct and medium-term application in society or in the productive sector.

For Hernández, Fernández and Baptista (2010)

con frecuencia, la meta del investigador consiste en describir fenómenos, situaciones, contextos y eventos; esto es, detallar como son y se manifiestan. Los estudios descriptivos buscan especificar las propiedades, las características y los perfiles de personas, grupos, comunidades, procesos, objetos o cualquier otro fenómeno que se someta a un análisis [the researcher often aims to describe phenomena, situations, contexts and events, that is, to detail what they are like and how they are shown. Descriptive studies seek to specify the properties, characteristics and profiles of people, groups, communities, processes, objects or any other phenomenon that is subjected to analysis]. (p. 80) 
As for the research design, it is non-experimental since the variable, the object of study, will not be manipulated.

Data collection instruments such as interviews and surveys were applied to the users of the Spring ERP system/cash register module.

In the case of the interviews, performance measurements and comparisons were applied to the Spring ERP system and the Msante system to determine the number of patients that a user could attend to in each case and thus determine the improvement with the ERP system.

In the case of the surveys, the benefits of implementing the Spring ERP system/cash register module were tested, corroborated, and demonstrated.

\section{RESULTS}

The following Tables and Figures represent the results obtained from the fieldwork of the independent variable applied to the universe of fifteen users of the cash register module, through interviews and surveys.

The unit of measurement used was the time (minutes) required by both the Spring ERP system and the Msante system to attend to a patient.

Also, to determine the number of patient services supported by each system, the answers obtained from the users interviewed were averaged. For example, in question 1 of the interview, the following was stated: "Has the Spring ERP system improved operations by reducing or eliminating manual work? How much time did you spend per patient in each system?"

Thus, the time needed per patient in each system was determined, which is reflected in Table 1.

It must be considered that an hour equals sixty minutes; therefore:

- $\quad$ Spring $=60 / 3.75=16$ patients per hour

- Msante $=60 / 22.50=2.66$ patients per hour

Man-hours during the week are also included, which are 12 work hours from Monday to Saturday. Sundays were not considered since they are not working days.

After applying these calculations, the following results are obtained:

- Total hours spent per cashier/user in a day = 12 hours

- Total working days $=26$ days

- Total hours worked in a month (26 days x 12 hours) $=312$ hours

Table 2 details the degree of efficiency according to the time it takes a user to run each system when attending to a patient; first, it shows the minutes it takes per patient, then, the number of patients attended per hour and per month.

The calculation per month is obtained after the following procedure:

Table 1. Time Spent by User in Each System per Patient.

\begin{tabular}{|l|c|c|}
\hline \multicolumn{1}{|c|}{ User } & Spring ERP System & Msante System \\
\hline User 1 & 4 minutes & 10 minutes \\
\hline User 2 & 3 minutes & 30 minutes \\
\hline User 3 & 3 minutes & 20 minutes \\
\hline User 4 & 5 minutes & 30 minutes \\
\hline Sum of time spent & 15 minutes & 100 minutes \\
\hline Average time spent per patient & 3.75 minutes & 22.50 minutes \\
\hline
\end{tabular}

Source: Prepared by the author.

Table 2. Patient Care Efficiency Analysis for Each System.

\begin{tabular}{|l|c|c|c|}
\hline \multicolumn{1}{|c|}{ System } & Minutes/patient & Patients/hour & Patients/month \\
\hline Spring ERP & 3.75 & 16.00 & 4992 \\
\hline Msante & 22.50 & 2.66 & 830 \\
\hline
\end{tabular}

Source. Prepared by the author. 
- Spring $=16$ patients $\times 312$ hours $=4992$ patients.

- Msante $=2.66$ patients $\times 312$ hours $=830$ patients.

Figure 1 shows the results of monthly services per user and per system. The current Spring ERP/cash register module shows an improvement in the work per cashier, since it increases its monthly throughput by more than 4000 patients, while the Msante system only allowed up to 830 patients per user.

The comparison shows a percentage increase of $501.45 \%$.

Based on interviews conducted, Table 3 shows the efficiency based on the time a user spent working in each system by examining the collection capacity per patient in minutes, hours, and month.

Figure 2 shows the throughput in the number of patients served that each system could offer per cashier in the cash register collection task. The ERP system improves the monthly service capacity by serving up to 6808 patients, while the previous system supported up to 2203 patients, which translates into a percentage increase of $209.03 \%$, as detailed below:

Likewise, when asked how this ERP system contributed to the control, the following results were obtained from the most experienced users, who established nine benefits of this system (see Figure 3 ).

The dependent variable was also analyzed to measure the productivity of each computer program, selecting four users of the cash module with knowledge of both systems and who remained in the clinic after the activation of the new system.

According to Argota and Argota (2018) "La prueba t-Student es una de las más utilizadas para la comparación de dos muestras en diferentes escenarios de interés [The $t$-Student test is one of the most commonly used tests to compare two samples in different scenarios of interest]" (p. 2).

The scenarios were determined based on the general hypothesis "The implementation of an ERP

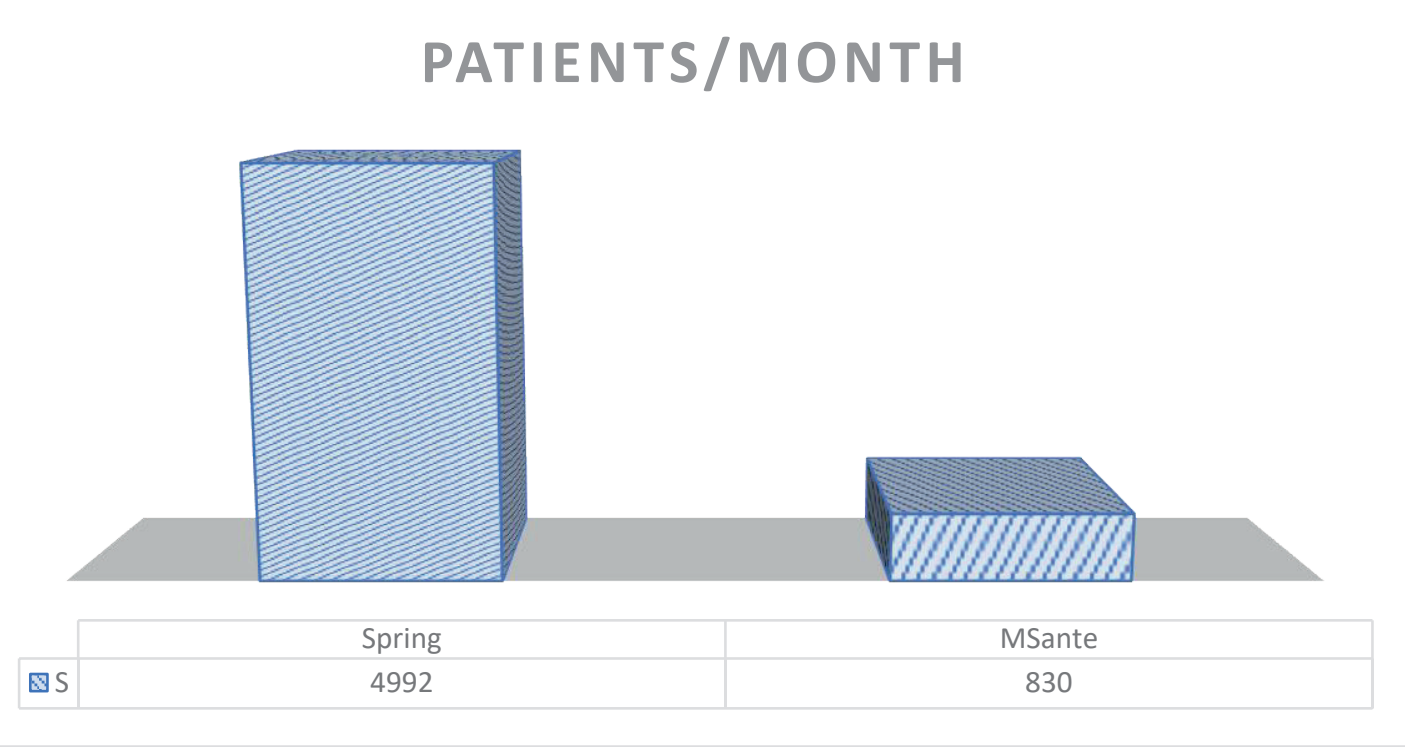

Figure 1. Performance analysis of each system with respect to the number of patients served per month.

Source: Prepared by the author.

Table 3. Analysis of Cash Register Collection Efficiency.

\begin{tabular}{|l|c|c|c|}
\hline \multicolumn{1}{|c|}{ System } & Minutes/patient & Patients/hour & Patients/month \\
\hline Spring & 2.75 & 21.82 & 6808 \\
\hline Msante & 8.50 & 7.06 & 2203 \\
\hline
\end{tabular}

Source: Prepared by the author. 


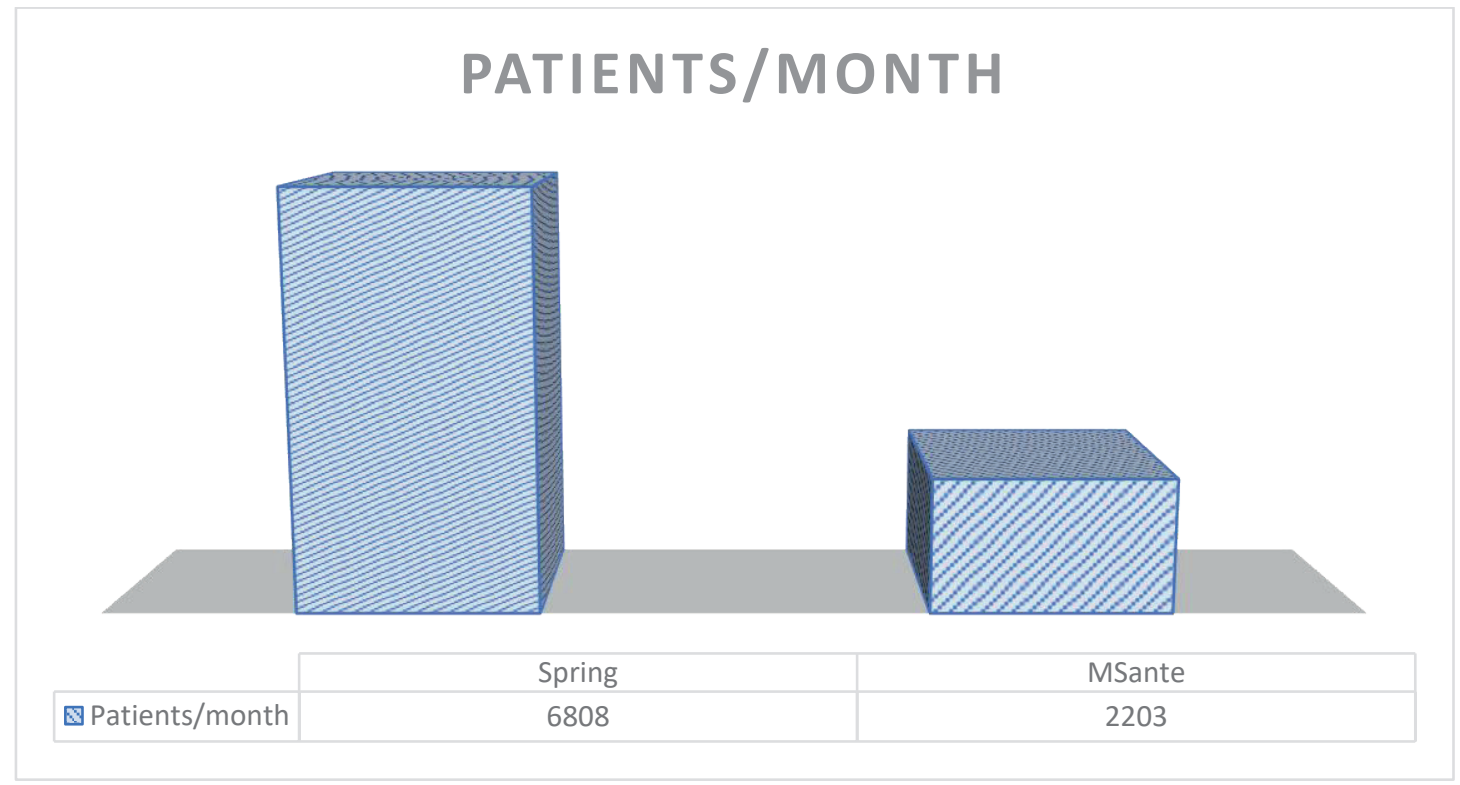

Figure 2. Throughput analysis of patient servings offered by each system per cashier in the cash collection task.

Source: Prepared by the author.

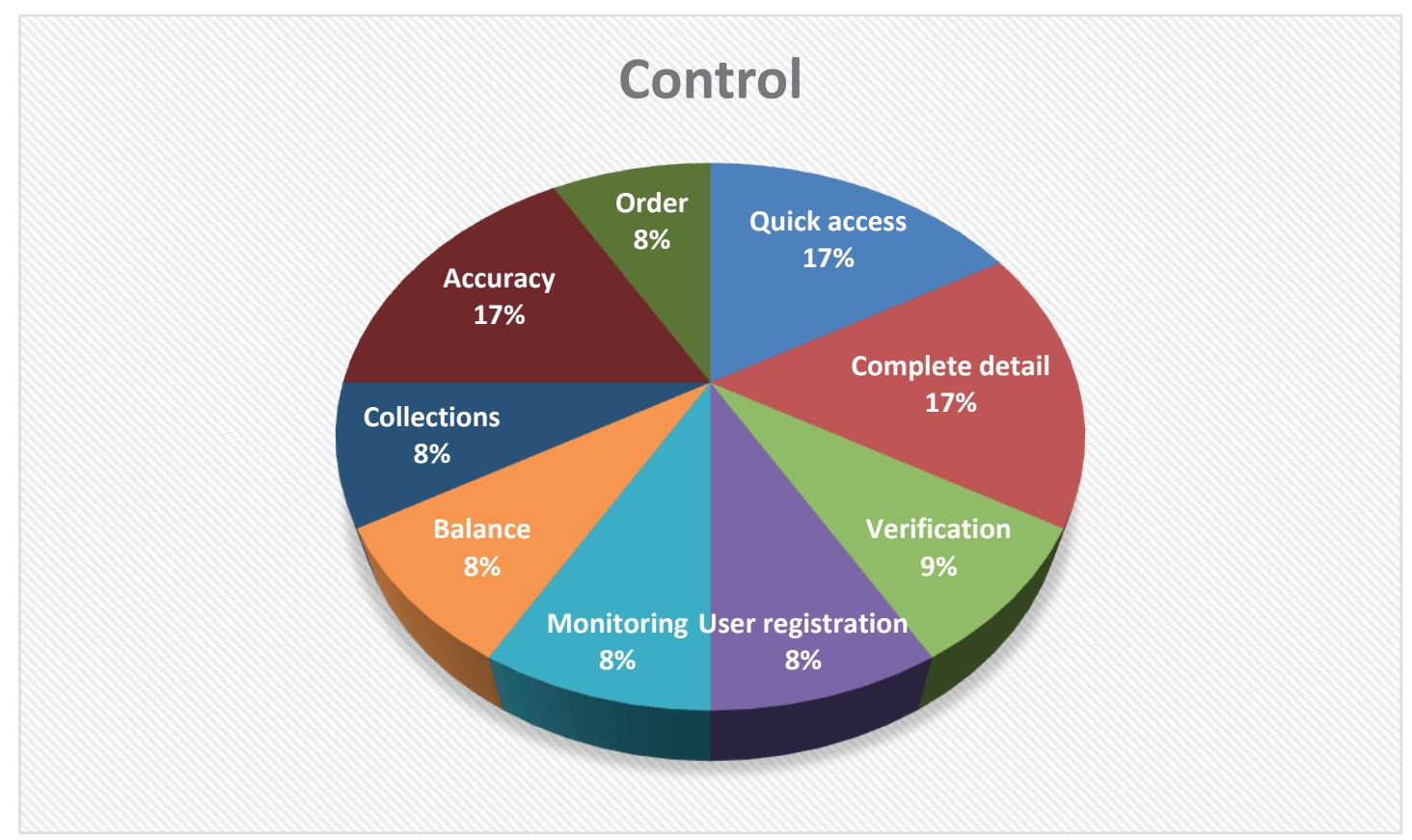

Figure 3. Analysis of control factors.

Source: Prepared by the author.

system improves the control of the cash register collections in an important clinic located in the city of Lima".

The hypothesis was tested by means of the t-Student test; the minutes spent in serving each patient were examined before and after the implementation, as shown in Table 4.
Where:

$\mathrm{H}_{0}=\mu \mathrm{d} \leq 0$

$\mathrm{H}_{1}=\mu \mathrm{d}>0$

After denoting:

$\mathrm{H}_{0}=$ Does not improve the control of cash register collections. 
$\mathrm{H}_{1}=$ Improves the control of cash register collections.

Also:

If $p$-valor $<\alpha \rightarrow \mathrm{H}_{0}$ is rejected.

If $p$-valor $\geq \alpha \rightarrow \mathrm{H}_{0}$ is accepted.

Based on the information in Table 4, the following results of the hypothesis testing analysis are obtained:

Calculation of the mean: $\overline{\mathrm{d}}=18.75$

Users: $\quad n=4$

Degree of freedom: $\quad g l=(n-1)=3$

Standard deviation: $\mathrm{Sd}=\sqrt{(d 1-d)^{\wedge} 2 / n-1}=$ 9.53502316

Test statistic: $\mathrm{t}=\frac{\overline{\mathrm{d}}}{\mathrm{Sd} / \sqrt{\mathrm{n}}}=3.93286931$

Critical value: $t(1-\alpha)(n-1)=2.35336343$

Significance level:

$\alpha=0.05 \rightarrow 5 \%$

$p$-value

0.01463716

Decision:The null hypothesis must not be used.

After analyzing the hypothesis test, it was verified that the test statistic is greater than the critical value and the $p$-value result is less than the $\alpha$ value; therefore, the null hypothesis $\left(\mathrm{H}_{0}\right)$ is rejected, and the alternative hypothesis $\left(\mathrm{H}_{1}\right)$ is accepted. In addition, it was observed that the mean is 18.75 , so the skewness is to the right.
Moreover, other types of analysis were taken in order to demonstrate the hypotheses, in which post-implementation improvements were corroborated by means of surveys conducted with ERP system/cash register module users, who gave positive responses regarding the implementation.

Measurement scales were established to determine the degree of acceptability; the results are shown in Table 5.

The general hypothesis was tested through surveys applied to 11 users of the cash register module. This test obtained an average score of 49 points out of 55 , representing $89 \%$, which showed that the implementation of the ERP system in the clinic is acceptable, as shown in Figure 4.

Additionally, to determine its degree of acceptability, the three specific hypotheses with their respective indicators were contrasted.

The first specific hypothesis elaborated was "The automation of operations by means of the Spring ERP system/cash register module will contribute to the control of cash register collections in an important clinic located in the city of Lima".

Eight indicators were established; the acceptability measure of each one exceeded $80 \%$, which resulted in the first specific hypothesis being accepted, as shown in Figure 5.

The second specific hypothesis elaborated was "The improvement of the information process by

Table 4. Analysis of Service Samples in Minutes per Patient.

\begin{tabular}{|c|c|c|c|}
\hline User & Pre implementation & Post implementation & $\overline{\mathrm{d}}$ \\
\hline 1 & 10 & 4 & 6 \\
\hline 2 & 30 & 3 & 27 \\
\hline 3 & 20 & 3 & 17 \\
\hline 4 & 30 & 5 & 25 \\
\hline
\end{tabular}

Source: Prepared by the author.Donde:

Table 5. Measurement Scores and Value Scale.

\begin{tabular}{|l|c|c|}
\hline \multicolumn{1}{|c|}{ Determination } & Score & Percentage Degree of Value \\
\hline Excellent & 5 & $81-100 \%$ \\
\hline Very good & 4 & $61-80 \%$ \\
\hline Good & 3 & $41-60 \%$ \\
\hline Fair & 2 & $21-40 \%$ \\
\hline Poor & 1 & $0-20 \%$ \\
\hline
\end{tabular}

Source: Prepared by the author. 
PERCENTAGE DEGREE OF ACCEPTABILITY VALUE

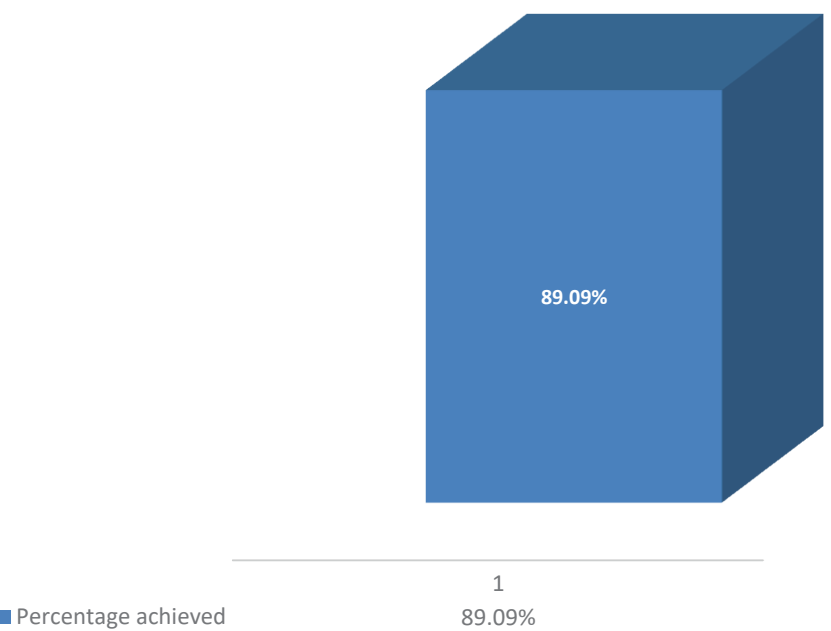

Figure 4. Acceptability of the general hypothesis. Source: Prepared by the author.

\section{PERCENTAGE DEGREE OF ACCEPTABILITY}

The cash register module allows to collect payment for an additional or non-booked medical appointment without any difficulty

The cash register module enables medical appointment booking even when a doctor's office is

$$
\text { not available }
$$

The cash register module shows the availability of doctors and offices for the payment collection of a booked medical appointment

The cash register module accurately reports the amount to be collected

The system allows for currency conversion in the issuance of vouchers for the customer
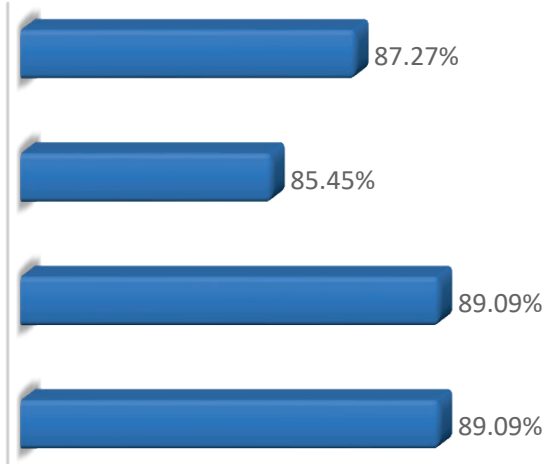

Issuance of payment vouchers to the customer is easy

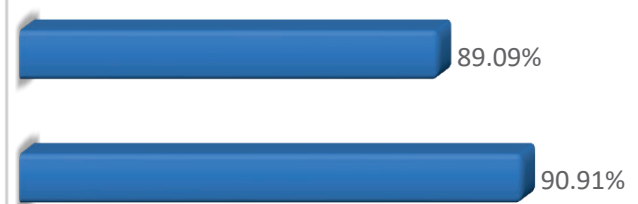

The cash register module makes work without trouble possible

The cash register module is user-friendly

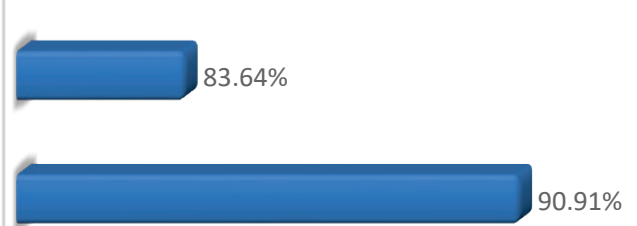

Figure 5. Acceptability indicators of the first specific hypothesis.

Source: Prepared by the author. 
means of the Spring ERP system/cash module will increase the control of cash collections in an important clinic located in the city of Lima".

Six indicators were established; the acceptability measure of each one exceeded $80 \%$, which resulted in the second specific hypothesis being accepted, as shown in Figure 6:

The third specific hypothesis elaborated was "The cash counts by means of the Spring ERP system/ cash register module will generate an adequate control of cash register collections in an important clinic located in the city of Lima".

Ten indicators were established; the acceptance measure of each one exceeded $80 \%$, which resulted in the third specific hypothesis being accepted, as shown in Figure 7:

\section{DISCUSSION}

Once the results of this research, obtained from field work through surveys and interviews, have been presented, it is corroborated that the implementation of an ERP system for the improvement of control has been successful, which is reflected in the degree of acceptability.

Undoubtedly, the use of the ERP system has resulted in significant changes in the clinic, since improvements were observed in maximizing productivity and a better support and service platform.

The automation, the improvement or ordering of the information processes and the various cash register reports for an adequate cash count have improved control and have been pleasantly received by the cash register users, since they have shown that the attention times have reduced, and users are able to complete their work at the established time.

\section{CONCLUSIONS}

The implementation of an ERP system in the clinic has given the expected results, as it has been demonstrated, since it improves aspects not only related to cash register collection control, but related to service times per patient, activities, and processes, since it is a system adaptable to the environment.

The implementation of the ERP system in the clinic has allowed to discover that this system offers a capacity or support platform for the maximum use of resources, since it is possible to optimize time and enhance the operation of users.

The automation of activities through the Spring ERP/ cash register module has proven to be user-friendly, allowing users to perform their tasks without difficulty, facilitating the issuance of payment vouchers and currency conversion and contributing to the ac-

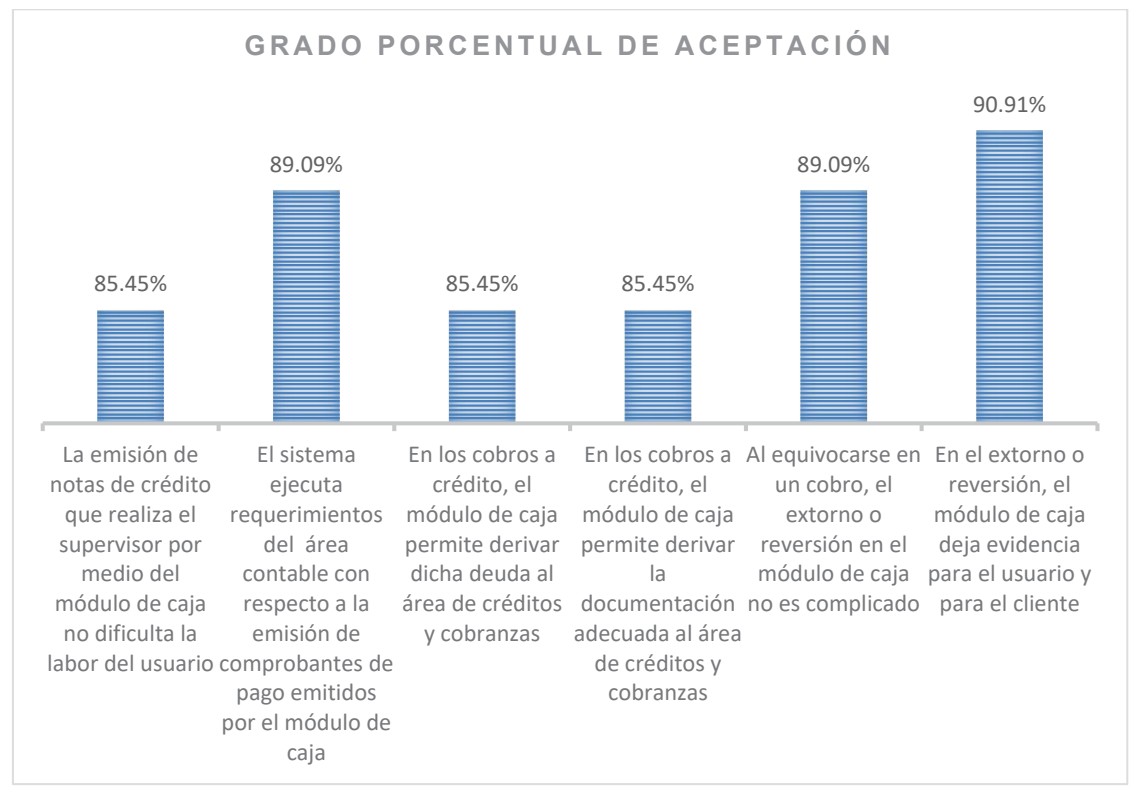

Figure 6. Acceptability indicators of the second specific hypothesis. Source: Prepared by the author. 


\section{PERCENTAGE DEGREE OF ACCEPTABILITY}

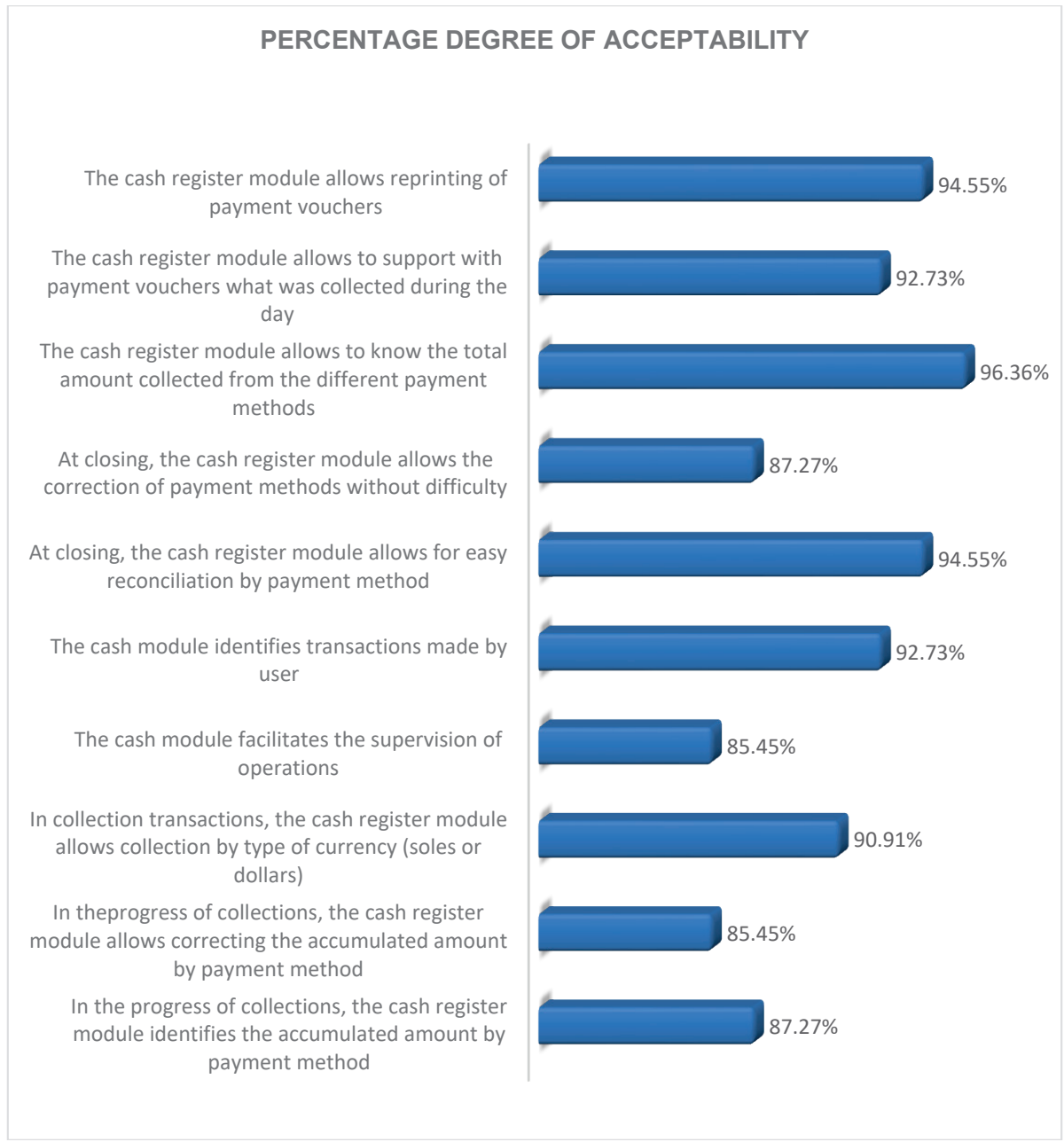

Figure 7. Acceptability indicators of the third specific hypothesis. Source: Prepared by the author.

curacy of collections; it also provides information on the availability of doctors and offices and enables the reservation and payment collection of medical appointments not booked on time.

The improvement of the information process through the Spring ERP system/cash register module makes it possible that the issuance of credit notes by supervisors does not hinder the work of the users; it has made it possible to avoid incidents with the accounting area; it makes it easier to transfer the documentation of a debt, or credit collection, to the credit and collection area; and it leaves evidence of transaction rebates.

The Spring ERP system/cash register module allows a better functioning of the progress of collections, since it allows the identification of the collected amount according to the payment method; it allows the correction of payment methods without difficulty; it facilitates the collection transactions by type of currency; it facilitates the cash supervision; and it allows that a reconciliation and presentation report is generated at cash closing. In addition, this system has contributed to improved cash reconciliation since it allows to adjust and correct payment methods. As for the issuance of the reconciliation reports, the system makes it possible to know the total collected by payment method, to adequately support each operation and to print vouchers that were not printed at the time of issuance.

\section{REFERENCES}

[1] Argota, G., \& Argota H. (2018). Aplicación de la prueba t-Student para la competencia técnica y trazabilidad analítica: ejemplo de estudio. Campus, 23 (26), 145-150. Retrieved 
from https://doi.org/10.24265/campus.2018. v23n26.04

[2] Duke, V., Navarro, M., Díaz, G., Pérez, Y., \& Vargas-Lombardo, M. (2016). Exploración en los sistemas CRM/ERP como estrategia en el sector PYMES. Revista de Iniciación Científica, 2(2), 86-94. Retrieved from https://revistas.utp. ac.pa/index.php/ric/article/view/1251/1414

[3] ESAN. (September 14, 2018). El sistema ERP en la gestión de proyectos. Retrieved from https://www.esan.edu.pe/apuntesempresariales/2018/09/el-sistema-erp-en-lagestion-de-proyectos/

[4] Farfán, J. (2020). La implementación de un sistema automatizado reduce los tiempos de atención en los procesos aplicables a la ventanilla única de turismo en la Municipalidad Provincial del Callao. Industrial Data, 23(2), 3137. Retrieved from http://dx.doi.org/10.15381/ idata.v23i2.15566

[5] Fernández, A. (2015). Gestión y Control administrativo de las operaciones de caja. Madrid, Spain: Editorial Elearning S.L.

[6] Guerrero, A., Marín, M., \& Bonilla, D. (2018). Erp como alternativa de eficiencia en la gestión financiera de las empresas. Revista Lasallista de Investigación, 15(2), 182-193. Retrieved from https://doi.org/10.22507/rli.v15n2a14

[7] Hernández, I., Báez, P., \& Córdova, J. (2018). Desarrollo de software de simulación ERP para herramienta didáctica en el proceso de enseñanza-aprendizaje. Revista de Tecnologías de la Información, 5(17), 1-8. Retrieved from https://www. ecorfan.org/bolivia/researchjournals/ Tecnologias_de_la_Informacion/vol5num17/ Revista_de_Tecnolog\%c3\%adas_de_la_ Informaci\%c3\%b3n_V5_N17_1.pdf

[8] Hernández, R., Fernández, C., \& Baptista, L. (2010). Metodología de La Investigación. México D.F, Mexico: McGraw-Hill/ Interamericana Editores S.A de CV.
[9] Hernández, J., Carreño, M., Sandoval, J., Estrada, I., \& Ignacio, R., (2016). Propuesta de criterios de evaluación en la implementación de ERP's en instituciones de educación superior. Pistas Educativas, 38(122), 232-243. Retrieved from http://itcelaya.edu.mx/ojs/index. php/pistas/article/view/679

[10] Hurtado, E., Arroyo, N., \& Guzmán, F. (2019). El Control interno y la importancia de su aplicación en las compañías. Revista Observatorio de la Economía Latinoamericana, (agosto 2019). Retrieved from https://www.eumed.net/rev/ oel/2019/08/control-interno-companias.html

[11] Lozada, J. (2014). Investigación aplicada: Definición, propiedad intelectual e industria. CienciAmérica 3(1), 34-39. Retrieved from http://cienciamerica.uti.edu.ec/openjournal/ index.php/uti/article/view/30

[12] Mayorga, M., Espinosa, M., López, A., \& Chango, M. (2020). Control interno para el área de cajas en Cooperativas de Ahorro y Crédito. Caso de estudio: Cooperativa de Ahorro y Crédito Indígenas Galápagos Ltda. Visionario Digital, 4(2), 57-80. Retrieved from https://doi. org/10.33262/visionariodigital.v4i2.1215

[13] Núñez, R. (2016). Software ERP. Análisis y Consultoría de Software Empresarial. Madrid, Spain: IT Campus Academy.

[14] Oltra Badenes, R. (2012). Sistemas Integrados de Gestión Empresarial. Evolución histórica y tendencias de futuro. Valencia, Spain: Universitat Politècnica de València.

[15] Riascos, S., \& Arias, V. (2016). Análisis del impacto organizacional en el proceso de implementación de los Sistemas de Información ERP - Caso de estudio. Entramado, 12(1), 284302. Retrieved from http://dx.doi.org/10.18041/ entramado.2016v12n1.23127

[16] Suárez, C. (2016). Guía Práctica. Software de gestión empresarial. Madrid, Spain: Edisa. 